\title{
Non Linear Flux Assessment Model for Endotoxin Neutralizing Pathways
}

Bharath BR, Manjunatha $\mathrm{H}^{*}$, Santosh Kumar HS and Bharath K

Department of P.G studies and Research in Biotechnology and Bioinformatics, Kuvempu University, Jnanasahyadri, Shankaraghatta-577 451, Shivamogga, Karnataka, India

\begin{abstract}
Graph drawing is one of the imperative techniques for understanding biological regulations in a cell or among cells at the pathway level. Dynamic modeling and simulation of signaling pathways is a fundamental issue in systems biology and has growing attention from researchers with experimental or theoretical background. Here is an attempt to develop a reliable method for the analysis and modeling of specific signaling pathways contributing for the endotoxin clearance. Here, integration of proteins in signaling pathways was analyzed, which enables the understanding of complex cellular processes. In pathway drawing, location information is essential for its comprehension. However, complex shapes need to be taken into account when torus-shaped location information such as nuclear inner membrane, nuclear outer membrane, and plasma membrane is considered. Schwartz introduced the notion of crosstalk, referring to the case that two inputs work through distinct signaling pathways but cooperate to regulate cell signaling. In disquiet to that current study hypothesized at adopting electronic circuit block diagrams for the analysis of pathways contribute for endotoxin neutralization, by developing a logical circuit models and simulate using Very High Speed Integrated Circuit Hardware Description Language (VHDL), here mathematical models are developed as a viaduct between pathway model and circuit model. This method is reliable since it helps calculating the flux at each node. The method establishes that the multiple signaling pathways do not have the additive effect but the signals will cancel at the converging points. The established method can be applied for any network modeling and calculation of signal flux from different nodes to nucleus, degree of error depends on pathway modeling since it is a manual process errors in modeling can reflect on method reliability.
\end{abstract}

Keywords: Pathway modeling; Systems biology; Crosstalk; Endotoxin; Flux

\section{Introduction}

The pathogenic capability of gram negative bacteria is often associated with certain components of gram negative cell walls in particular, the lipopolysaccharide (LPS) layer. In humans, LPS triggers an innate immune response characterized by cytokine production and immune system activation [1-3]. Generally in animal intestine, millions of gram-negative bacteria are present and a very small level of endotoxin associated with these bacteria is also cleaned/ neutralized by liver, but once the level of endotoxin increases it may reach blood streams and spread to various parts of the body. If this spreading of endotoxin starts in body, the body immune system releases inflammatory substance and increases the body temperature and leads to sepsis or septic shock. If the presence of endotoxin inside the body is below the normal level, human immune system can fight back, then in such case destruction and inflammation of tissues at a very small level is the only harm the infection causes, but if the level of endotoxin is higher than the normal, then the infection can be life-threatening. The effect of the infection depends on the amount of endotoxin present inside the host body. The spread of endotoxin inside the host body and inflammatory reaction by the immune system is called endotoxic shock [4].

Endotoxins are in large part responsible for the dramatic clinical manifestations of infections with pathogenic Gram-negative bacteria, such as Neisseria meningitidis, the pathogens that cause meningococcal disease, including meningococcemia, Water house-Friderichsen syndrome, and meningitis [5].

Various researchers reported that the pathways like Nuclear FactorKappaB (NfkB) pathway [6,7], B-cell receptor [8], Tumor necrosis factor-alpha (TNF- $\alpha$ ) pathway $[9,10]$, Toll like receptor-4 (TLR4) pathway [11-13] and Jak-Stat pathway [7] are crucially causative for endotoxin neutralization, assessment of the interaction between these pathways itself is a big challenge infront of researchers. Several modelling schemes have been touted as suitable representations for pathways [14]. Ordinary and partial differential equations have proven to be very successful in metabolomics $[15,16]$. The key advantage afforded by logic modelling is the potential for accelerating hypothesis driven research and on this basis, it could change the success rate of new drugs in the market and the development of new therapeutic regimes. Indeed, industry has learnt over the years that the identification of a target for drug intervention does not necessarily equate with understanding what the target does. The lack of a more complete appreciation of the target biology has often been the underlying reason for complications resulting from adverse drug reactions [14].

At first glance, signaling can be seen as a linear connection between input elements (the receptors) and output elements (such as regulators of gene expression). A closer assessment reveals that signaling pathways interact with each other, forming a network [17]. To increase the degree of pathway assesment, Systems Biology Markup Language (SBML) [18] was created by the Systems Biology Workbench Development group in cooperation with representatives from many system and tool developers with in bioinformatics. By employing that the current study made an attempt towards the construction pathway models which can

*Corresponding author: Manjunatha H, Department of P.G studies and Research in Biotechnology and Bioinformatics, Kuvempu University, Jnanasahyadri, Shankaraghatta-577 451, Shivamogga, Karnataka, India, Tel: +91 8105580506 ; Fax: 08282-256255; E-mail: manjunatha75@gmail.com

Received November 19, 2012; Accepted March 16, 2013; Published March 20 , 2013

Citation: Bharath BR, Manjunatha H, Santosh Kumar HS, Bharath K (2013) Non Linear Flux Assessment Model for Endotoxin Neutralizing Pathways. J Comput Sci Syst Biol 6: 057-072. doi:10.4172/jcsb.1000101

Copyright: @ 2013 Bharath BR, et al. This is an open-access article distributed under the terms of the Creative Commons Attribution License,which permits unrestricted use, distribution, and reproduction in any medium, provided the original author and source are credited. 
use as tools for the dissection of complexity in understanding biological signaling pathways participating in endotoxic shock.

\section{Materials and Methods}

\section{Identifying the participation of genes in signaling pathways, construction and analysis of pathways}

KEGG (Kyoto encyclopedia of genes and genomes) database (http://www.genome.jp/kegg/pathway.html) was employed to identify the participation of genes in signaling pathways [19]. KEGG is a collection of online databases dealing with genomes, enzymatic pathways, and biological chemicals. The pathway databases records networks of molecular interactions in the cells and variants of them specific to particular organisms.

PANTHER Pathway database (http://www.pantherdb.org/) consists of over 165 primarily signaling pathways, each with subfamilies and protein sequences mapped to individual pathway components. The database was used to download signaling pathways involved in LPS neutralization like B-cell activation (PANTHER ID: P00010), TLR signaling pathway (PANTHER ID: P00054), TGF beta signaling (PANTHER ID: P00052), JAK-STAT signaling pathway (PANTHER ID: P00038) and WNT signaling pathway (PANTHER ID: P00057) in “.sbml” format and analyzed using CellDesigner 4.0.1. CellDesigner is a structural diagram editor for drawing gene regulatory and biochemical networks. We can browse and modify existing SBML models with references to existing databases, simulate and view the dynamics through an interactive graphical interface. So in present work, the tool was used to visualize, analyze and to merge the signaling pathways involved in endotoxic shock.

\section{Construction of mathematical models and electronic circuit block diagram for all the pathway nodes}

An initial model is constructed based on Protein-Protein Interactions (PPIs) in signaling pathway. Pathway model is constructed by taking the initial protein as $\mathrm{X}$ with its Value 1 and the next coming paths as $\mathrm{x}, \mathrm{y}$ and $\mathrm{z}$, the steps coming under $\mathrm{x}$ paths were labeled as $\mathrm{x}_{1}$, $\mathrm{x}_{2} \ldots \mathrm{x}_{\mathrm{n}}$ respectively, similarly for $\mathrm{y}$ and $\mathrm{z}$ paths were labeled. Here at the time of protein deviation through different paths, the protein value is divided as per the number of deviating paths. If the value at the protein is 1 and this protein is getting diverge through two paths, the value is shared as $1 / 2$ and $1 / 2$ to each path, if the value at the protein is 1 and this protein is getting diverge through three paths, the value is shared as $1 / 3$ and $1 / 3$ to each path and similarly, if the value at the protein is $1 / 2$ and this protein is getting diverge through two paths, the value was shared as $1 / 4$ and $1 / 4$ to each path. By following this principle, pathway models were drawn for all the five signaling pathways and merged signaling pathway network.

First problems arise already at this stage: links can be missing or discrepancies between model outcome and biological observation can arise. Resolving them requires new experimental data. The points that are not sufficiently defined in the network can also be analyzed in a computational approach that clarifies which of several alternative model assumptions may explain the observations. The initial model is then used to make predictions. These predictions are tested in new experiments or against new data for different mutant organisms or for different pathway stimulation scenarios. The results allow for a model update and a second round of iteration by taking the number of steps into consideration. This point is nicely illustrated by the development of mathematical cell models. Here, if pathway model have single step, it was labeled as $\mathrm{Y}$, if it's with two steps, it was labeled as $\mathrm{Y}^{2}$ and similarly for three and four steps it was labeled as $\mathrm{Y}^{3}$ and $\mathrm{Y}^{4}$ respectively. Here also, score sharing was same as in construction of pathway models and calculate the each models with an mathematical expressions get the value for all the models.

Later these mathematical cell models for all the pathways including merged network were converted into the block diagrams or circuit diagrams using the VHDL to describe a logic circuit by function, data flow behavior or structure. These circuits and mathematical cell models helped us to calculate the total flux on the nucleus by deriving the mathematical expressions.

\section{Results}

Signaling pathways in SBML $\left(^{*} . \mathrm{xml}\right)$ format were downloaded from PANTHER pathway database and visualized using CellDesigner 4.0.1 as in figures 1-5. All the five pathways merged together by using the CellDesigner 4.0.1 as shown in figure 6, here the common genes were identified in order to reveal the role of all the potential paths in signalling pathway for crosstalk.

The merging of the pathways is carried out by keen observation of each and every pathway and common signals in pathways are identified and merged as shown in figures 7-10. In B-Cell signaling pathway, IkB kinase (IKK) activation reaction upon phosphorylation is catalyzed by activated Protein kinase C (PKC), activated IKK further catalyses the dissociation of Ikappa B and NF- $\kappa B$ complex in to NF$\kappa \mathrm{B}$ and phoshorylated Ikappa $\mathrm{B}$ units and signals for NF- $\kappa \mathrm{B}$ as shown in figure 7A similarly in Toll like receptor (TLR) signaling pathway, transforming growth factor $\beta$ activated kinase-1 (TAK1) catalyses the IKK activation; here it activates IKK by phosphorylation. This activated IKK catalyses the dissociation of kappa B and NF-kappa B in to NFKappa B and phoshorylated Ikappa B units. The NF- kappa B continues its journey into nucleus as shown in figure 7B. We observed the same reactions in B-cell signalling, so this has been identified as a crosstalk region between TLR and B-cell signalling pathways as shown in figure 7C.

In TLR pathway, the activated kinase enzyme TAK1 catalyses the phosphorylation of another kinase enzyme MAP kinase kinase-3 (MKK3), phosphorilated MKK3 will get activate and act as a catalyst for the phosphorilation of c-Jun N-terminal kinases (JNK) and P38 to make them active, these couple of activated enzymes catalyses the activation of Activator Protein 1(AP1) by phosphorilation. This activated AP1 takes its journey into nucleus after phosphorylation as shown in figure 8A, likely in TGF signalling pathway as shown in figure $8 \mathrm{~B}$. TAK activation is observed due to phosphorylation and the reaction is catalyzed by Transforming growth factor beta- $\beta$ TGF $\beta$, TGF $\beta$ receptor I and TGF $\beta$ receptor II complex. The activated TAK will get combine with TGF-beta activated kinase 1/MAP3K7 binding protein $(\mathrm{TAB})$ and gives activated $\mathrm{TAK}$ and $\mathrm{TAB}$ heterodimer complex. The activated TAK and TAB heterodimer complex catalyses the P38 and JNK activation by phosphorylation. Then these activated P38 and JNK extends their journey in to nucleus. The same interactions were observed in TLR signalling pathway. The common signal was merged as shown in figure $8 \mathrm{C}$.

The TAK and TAB complex reacts with P38 and JNK and then converts activated phoshorylated P38 and JNK dimer which catalyses gene transcription. The activated STAT is detached from the heterotrimer complex composed of cytokine and activated JAK 


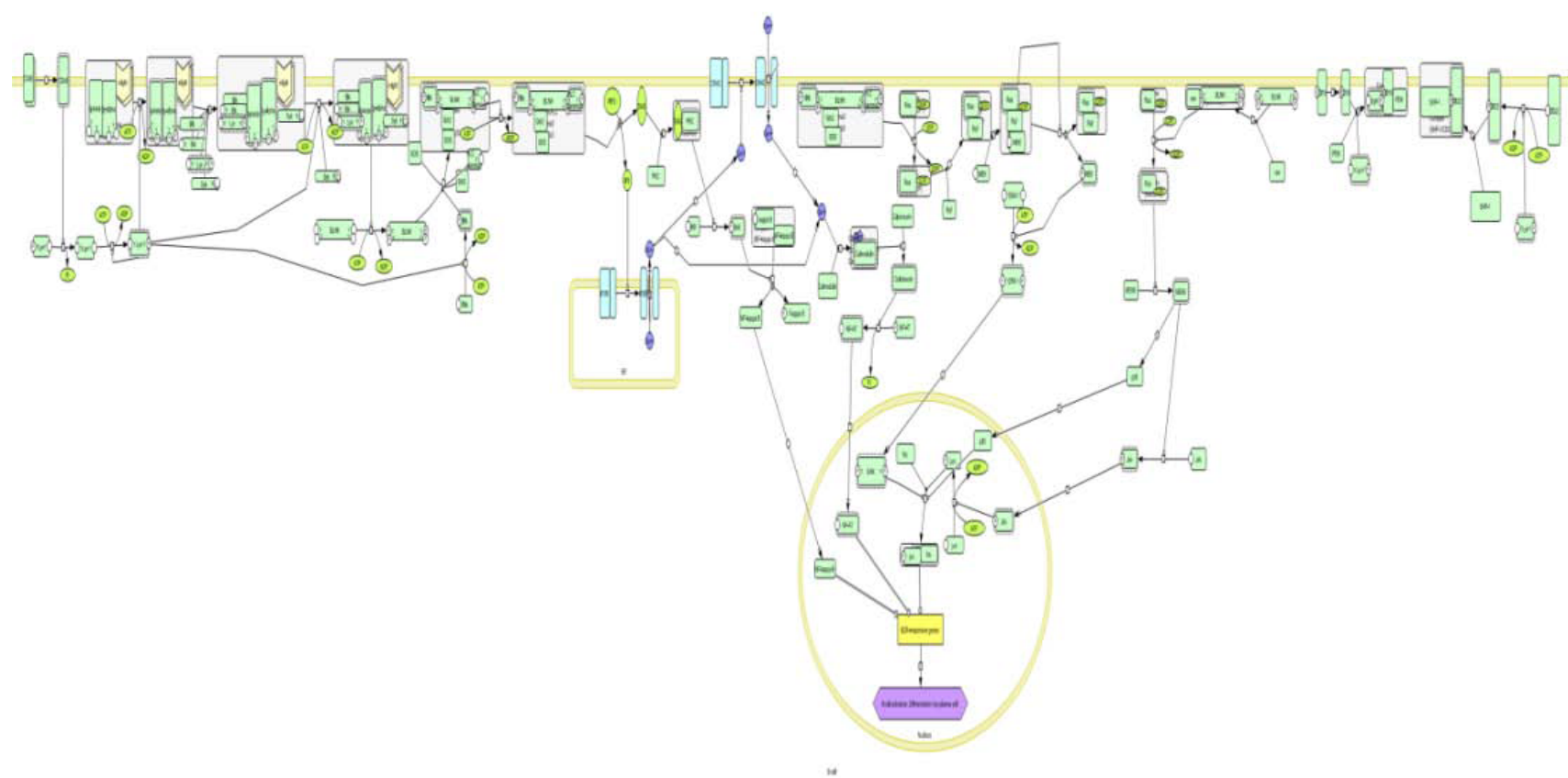

Figure 1: B-Cell signaling pathway edited and visualized using CellDesigner.

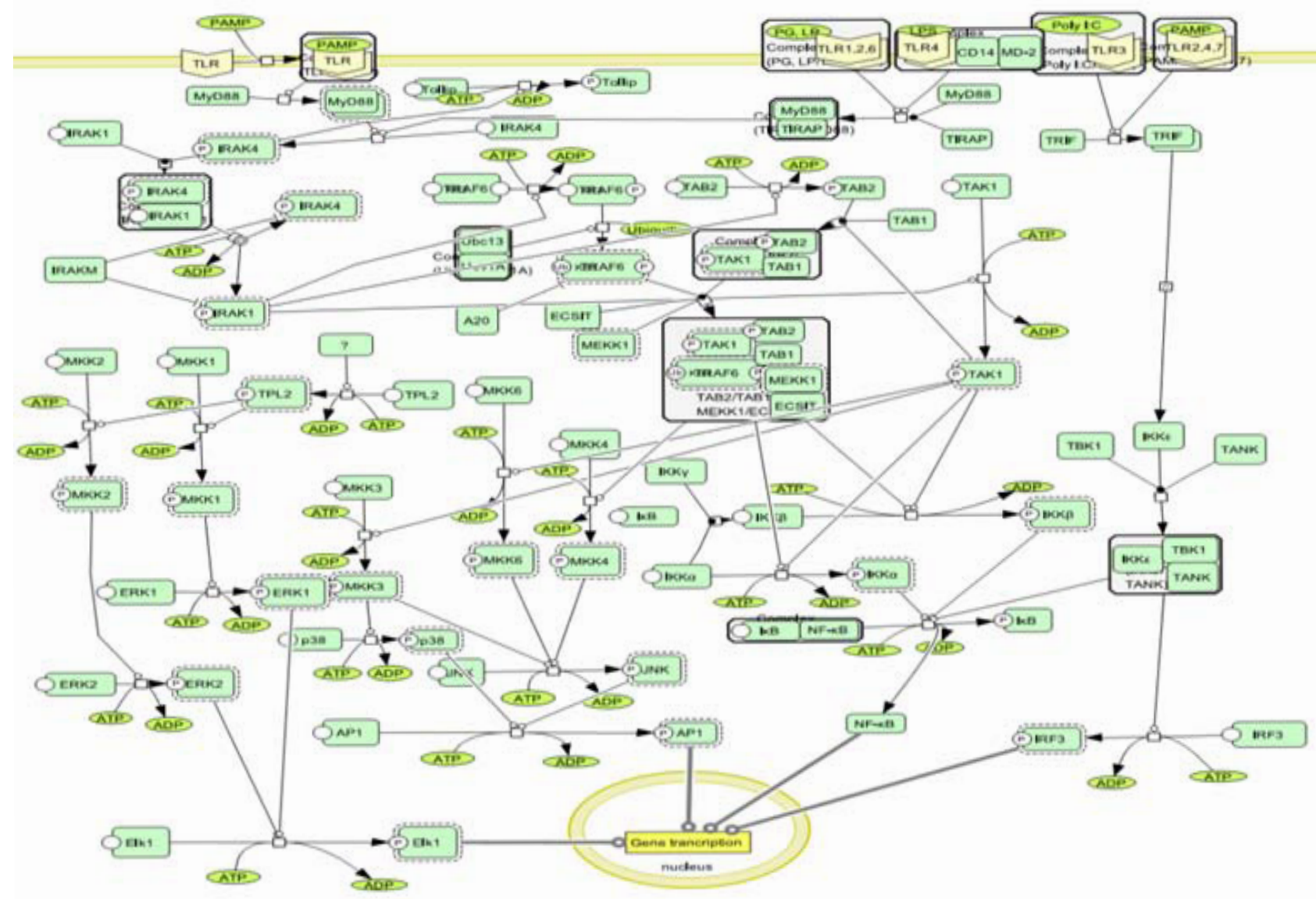

Figure 2: TLR signaling pathway edited and visualized using CellDesigner. 


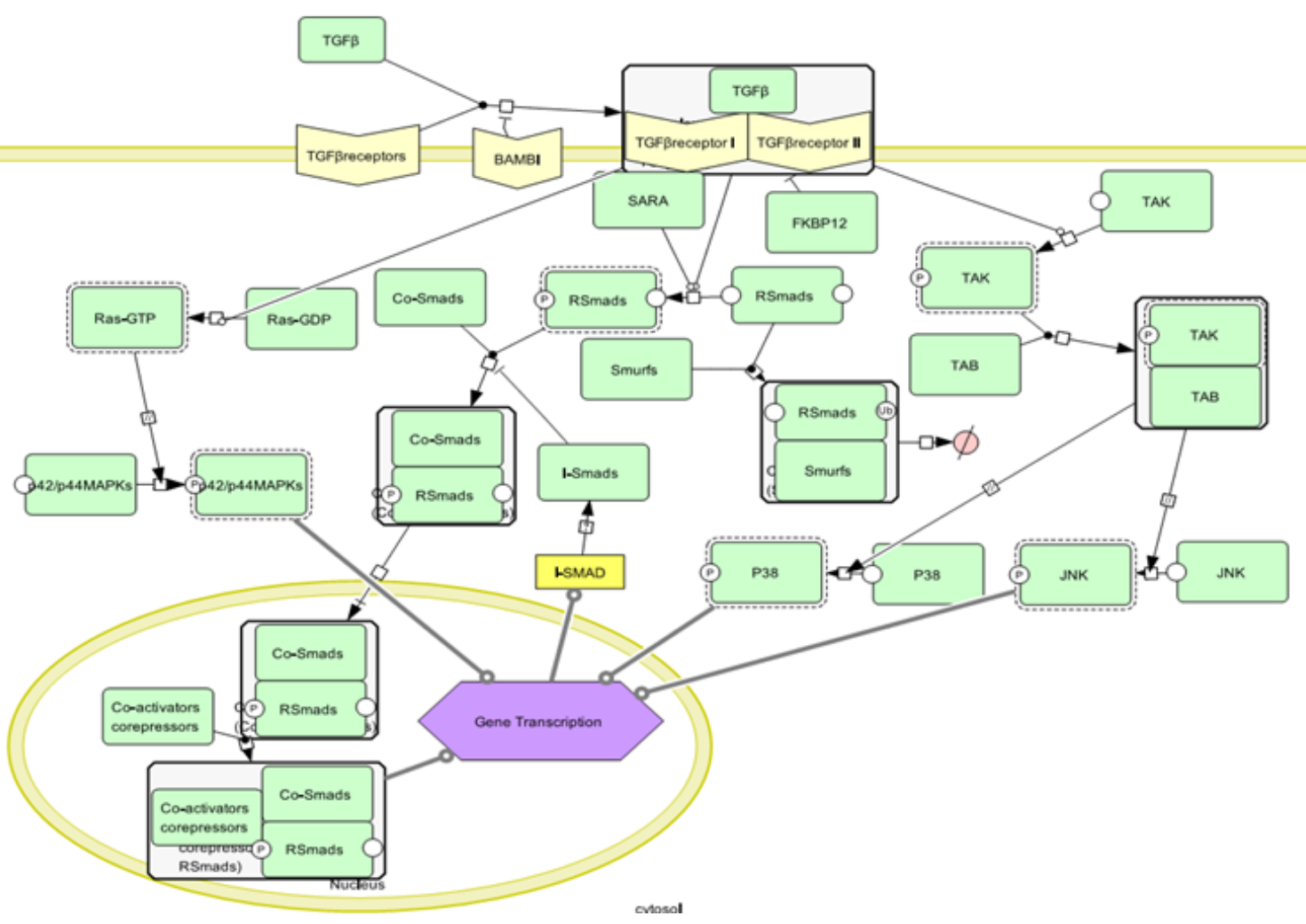

Figure 3: TGF beta signaling pathway edited and visualized using CellDesigner..

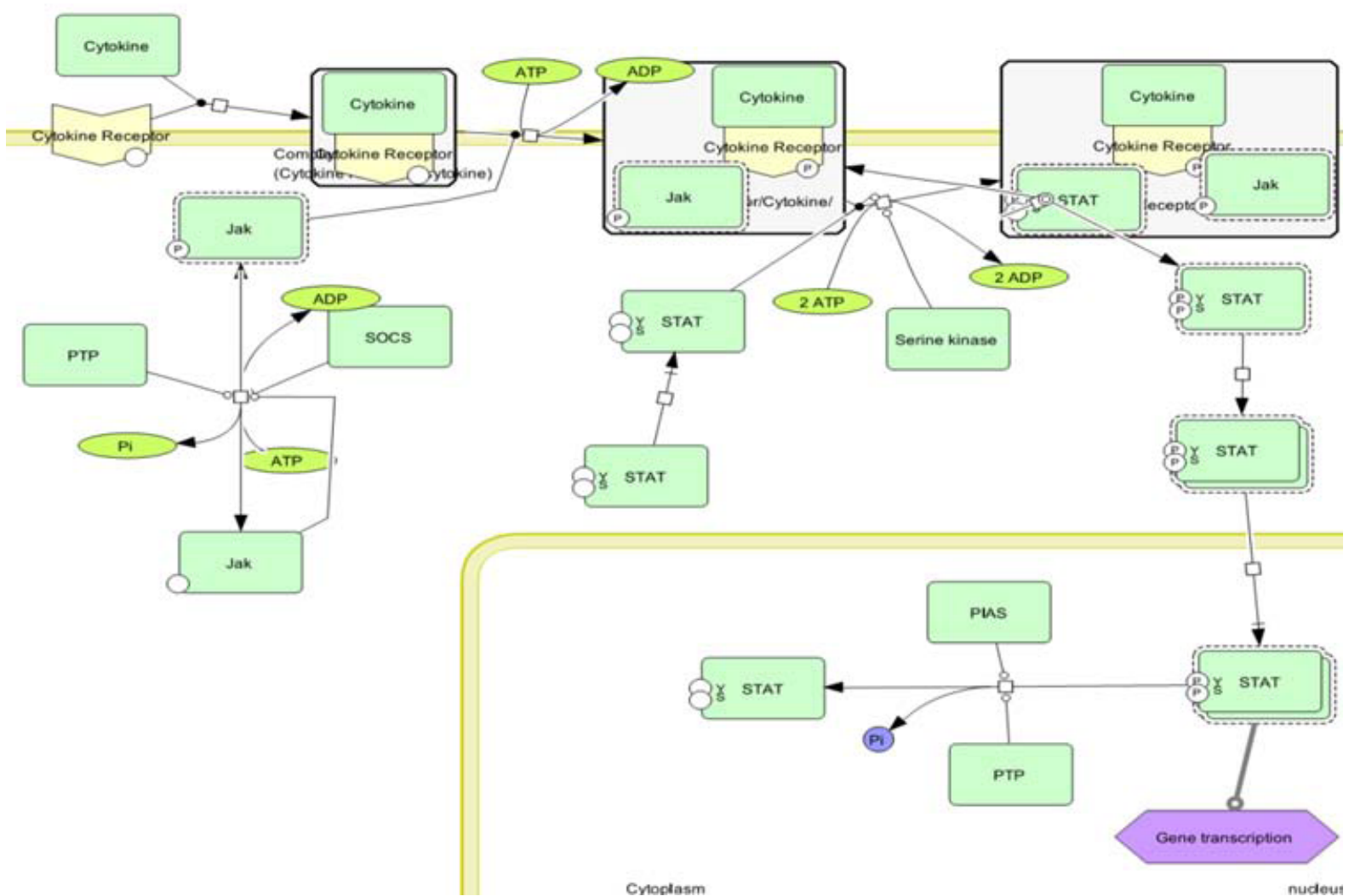

Figure 4: JAK-STAT signaling pathway edited and visualized using CellDesigner. 


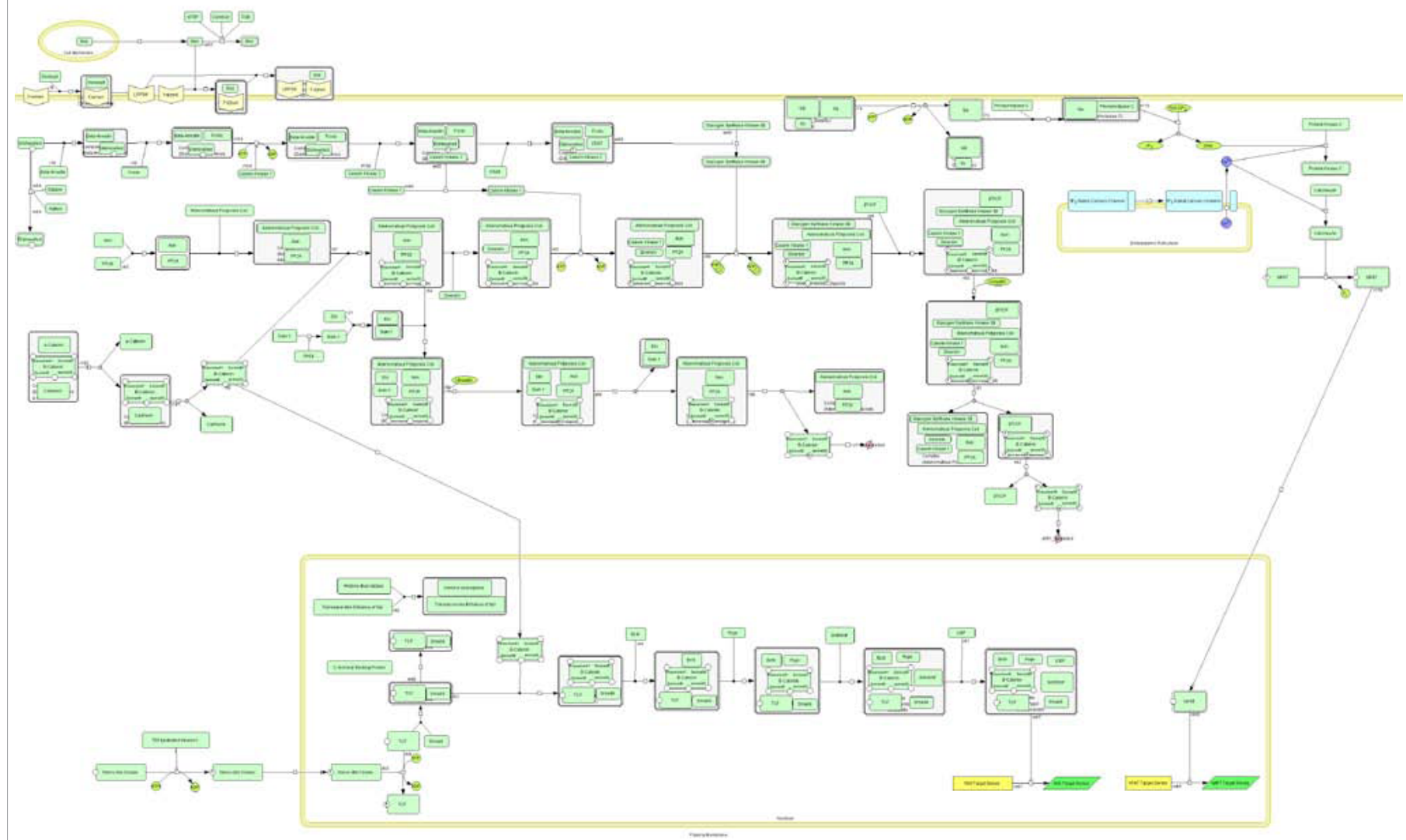

Figure 5: WNT signaling pathway edited and visualized using CellDesigner.

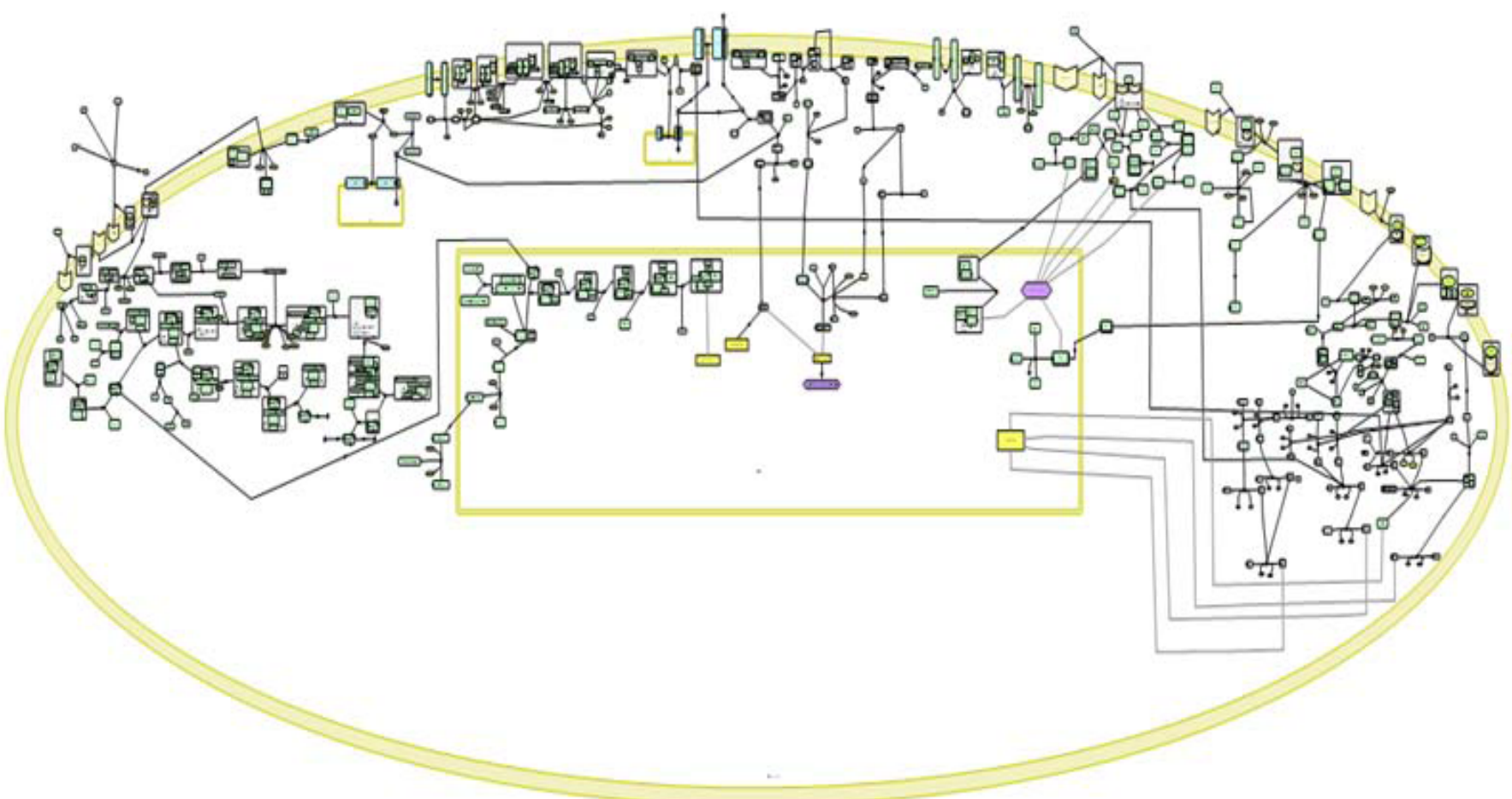

Figure 6: All the five signaling pathways merged and visualized using CellDesigner. 


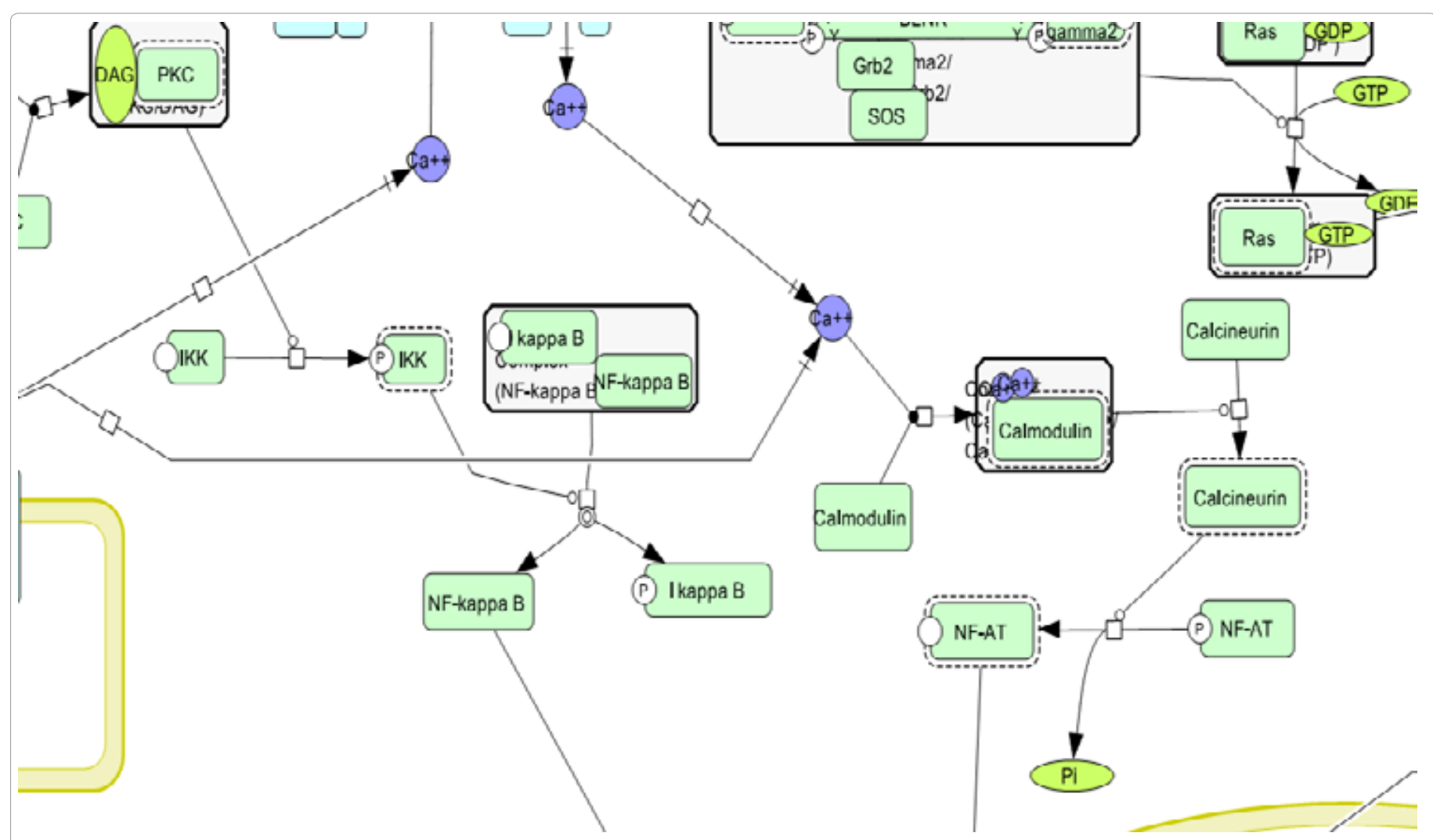

Figure 7a: B-Cell biological signaling pathways visualized in CellDesigner shows NF-kappa B signaling through IKK activation.

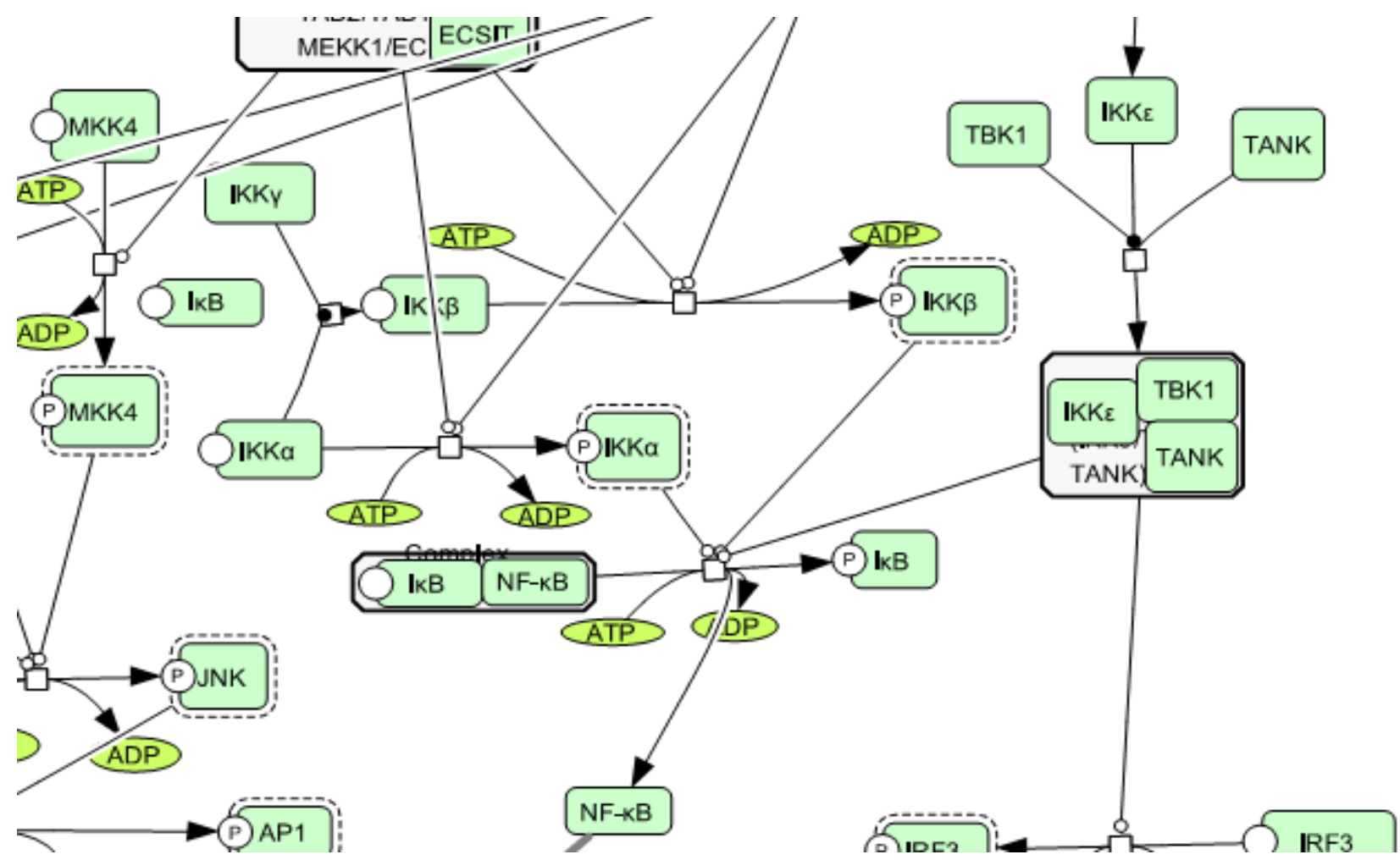

Figure 7b: TLR biological signaling pathways visualized in CellDesigner shows NF-KB signaling through IKK activation. 


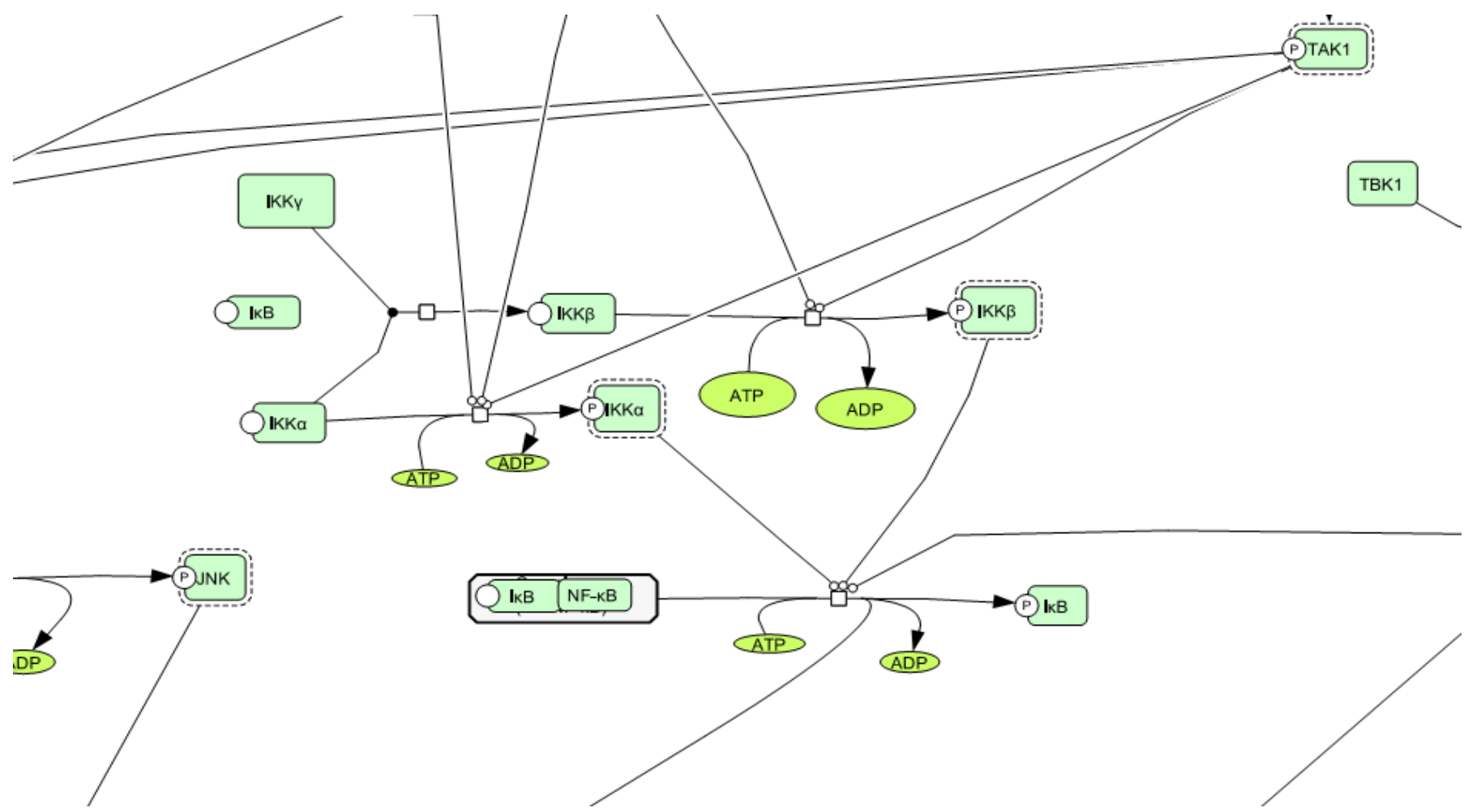

Figure 7c: Crosstalk between B-Cell and TLR biological signaling pathways visualized in CellDesigner.

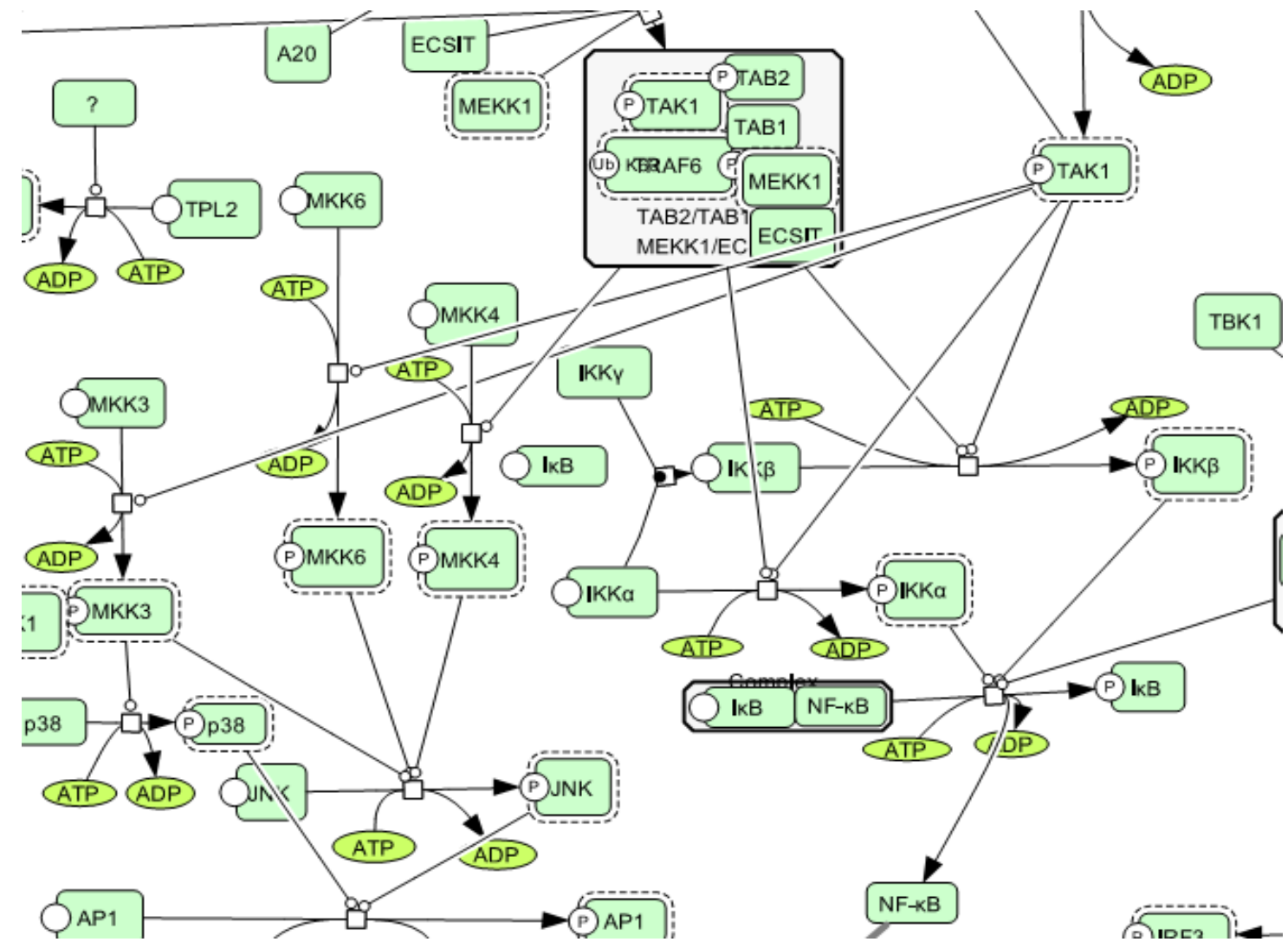

Figure 8a: TLR biological signaling pathways visualized in CellDesigner shows AP1 activation through TAK1, MKK3, JNK and P38. 


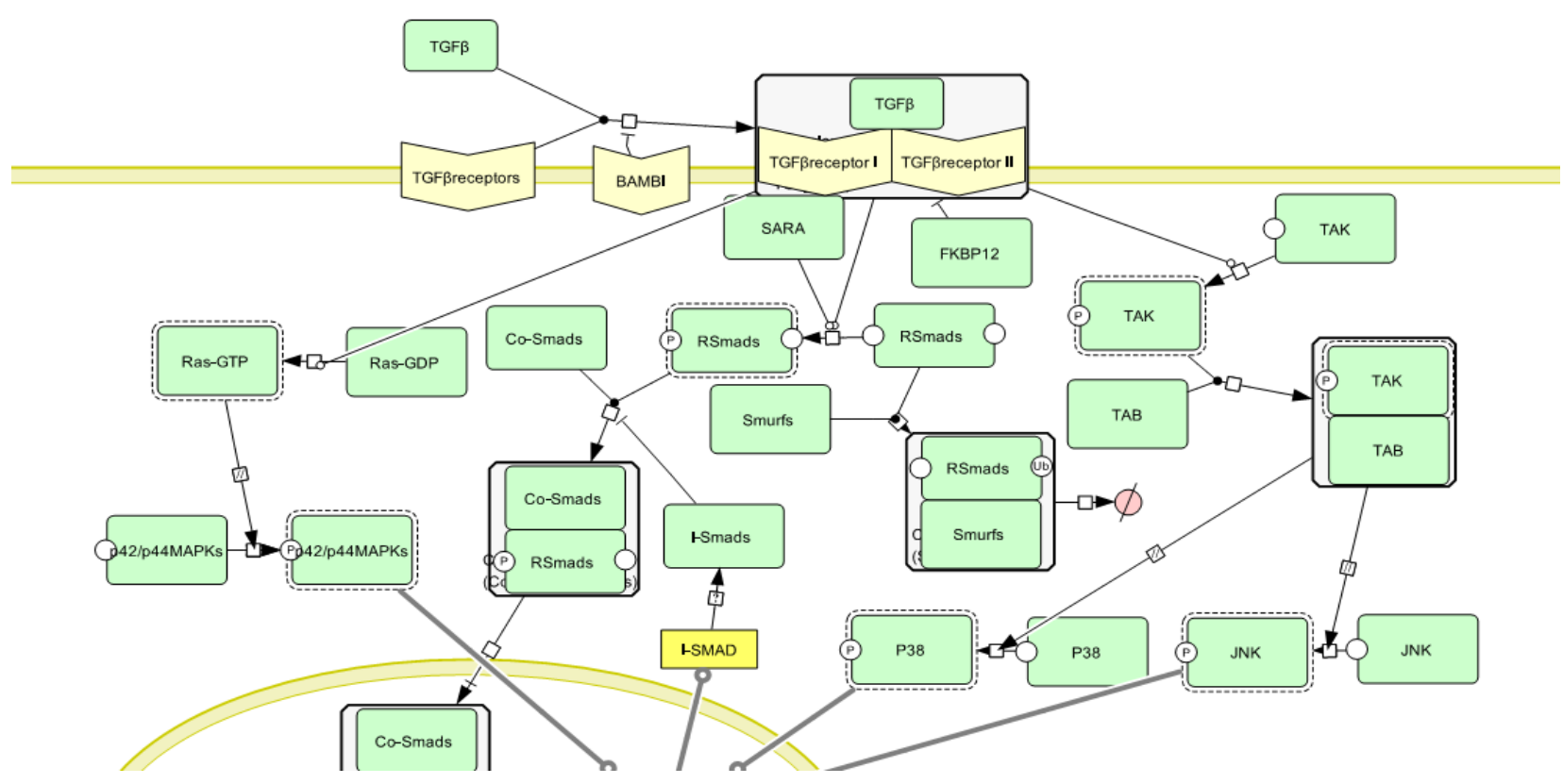

Figure 8b: TGF biological signaling pathways visualized in CellDesigner shows activation of P38 and JNK through TAK and TAB signals.

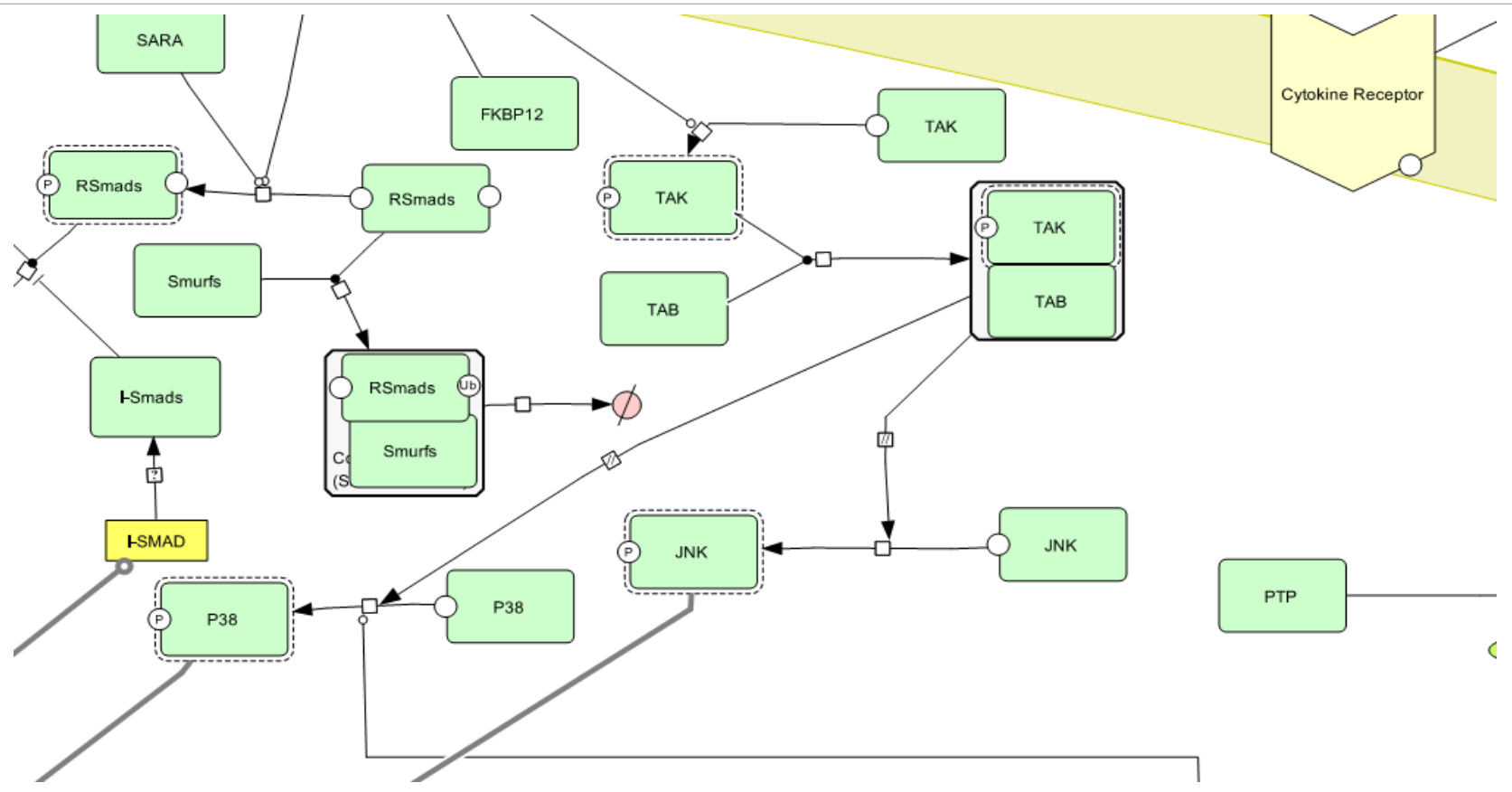

Figure 8c: Crosstalk between TLR and TGF biological signaling pathways visualized in CellDesigner.

and STAT. The detached and double phosphorilated STAT enters the nucleus and catalyses gene transcription.

In both TGF shown in figure 9A and JAK-STAT shown in figure $9 \mathrm{~B}$, pathways commonly linked at the region of gene transcription as shown in figure 9C. In B-Cell and WNT signals, binding of $\mathrm{Ca}^{2+}$ ion activates the calmodulin and catalyses the activation of calcineurin, the activated calcineurin catalyses the dephosphorylation of Nuclear factor of activated T-cells (NF-AT). The deprhosphorylated NF-AT will become active and enters the nucleus to catalyze the gene transcription.
In WNT pathway, calcineurin converted in to activated calcineurin. The activated calcineurin catalyzed in to phoshorylated NFAT then dephosphorylated to form activated NFAT.

In both B-Cell shown in figure 10A and WNT signaling pathways shown in figure 10B, the activation of NF-AT is common by dephosphorylation and the activation is catalyzed by the same enzyme called calcineurin, the path is recognized as common path for B-Cell activation and WNT signalling as shown in figure 10C. Structuring of pathway model is followed by the pathway analysis using CellDesigner 


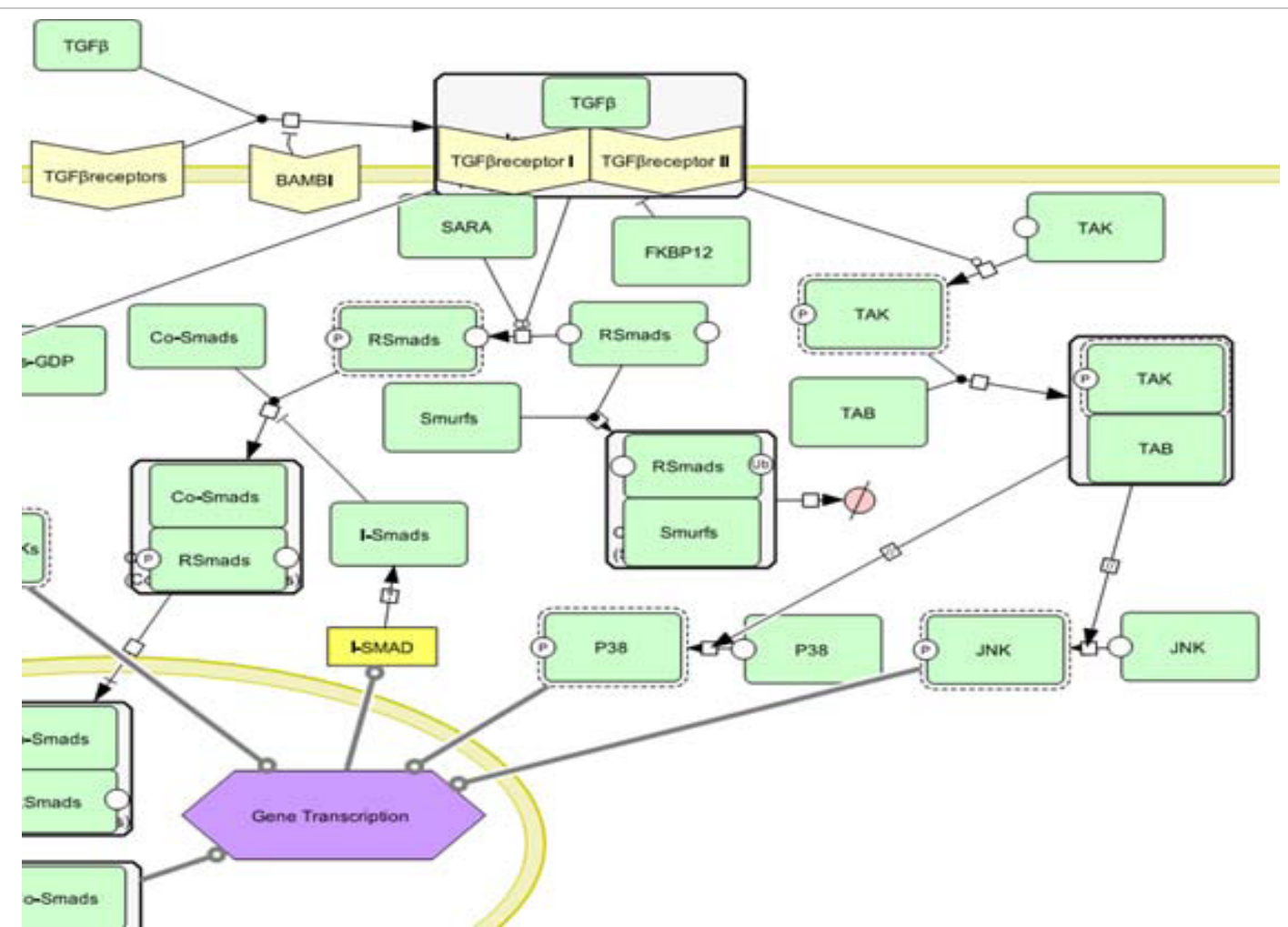

Figure 9a: TGF biological signaling pathways visualized in CellDesigner shows activation of p38 and JNK and leading to gene transcription

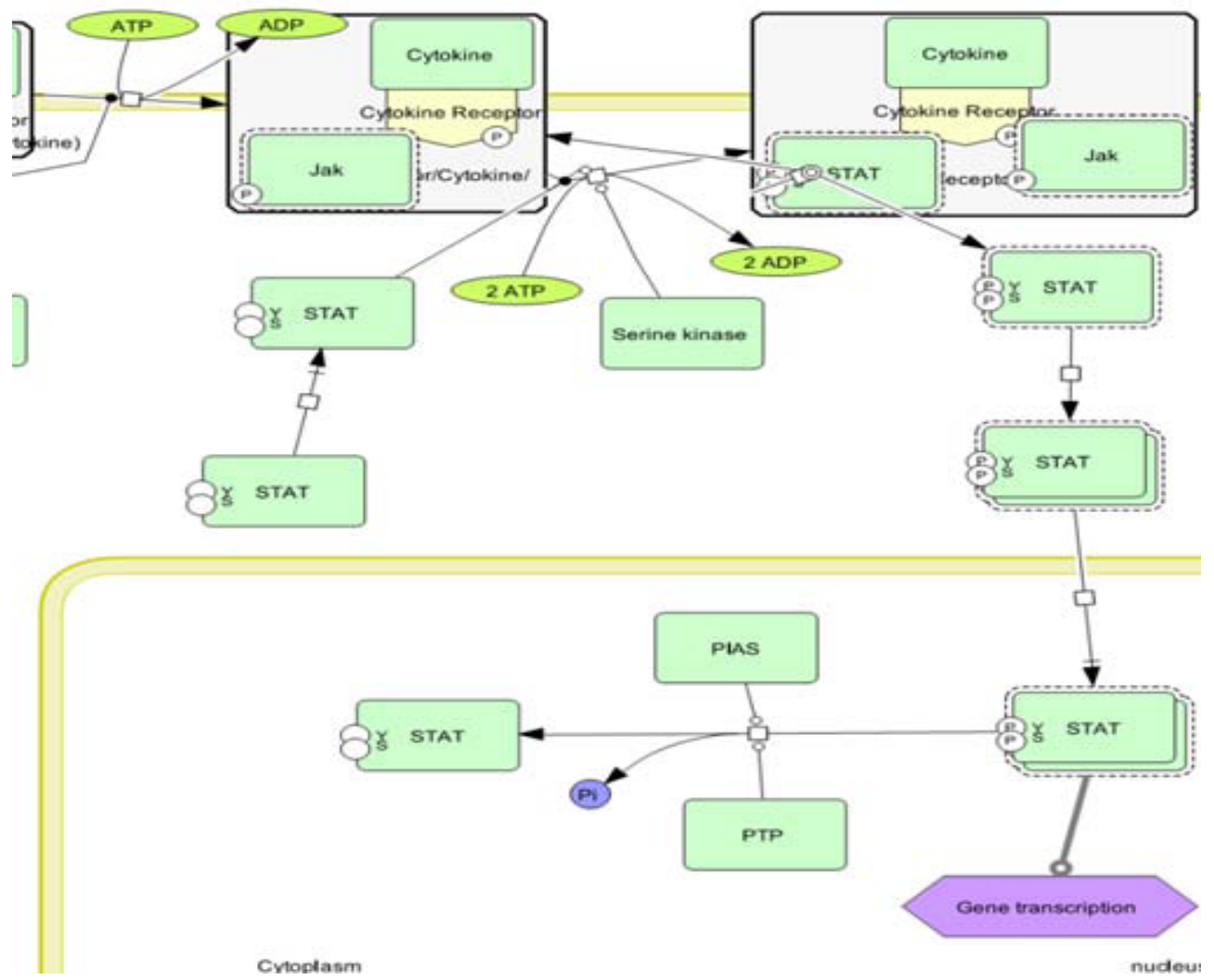

Figure 9b: JAK-STAT biological signaling pathways visualized in CellDesigner shows activated STAT signaling for transcription. 


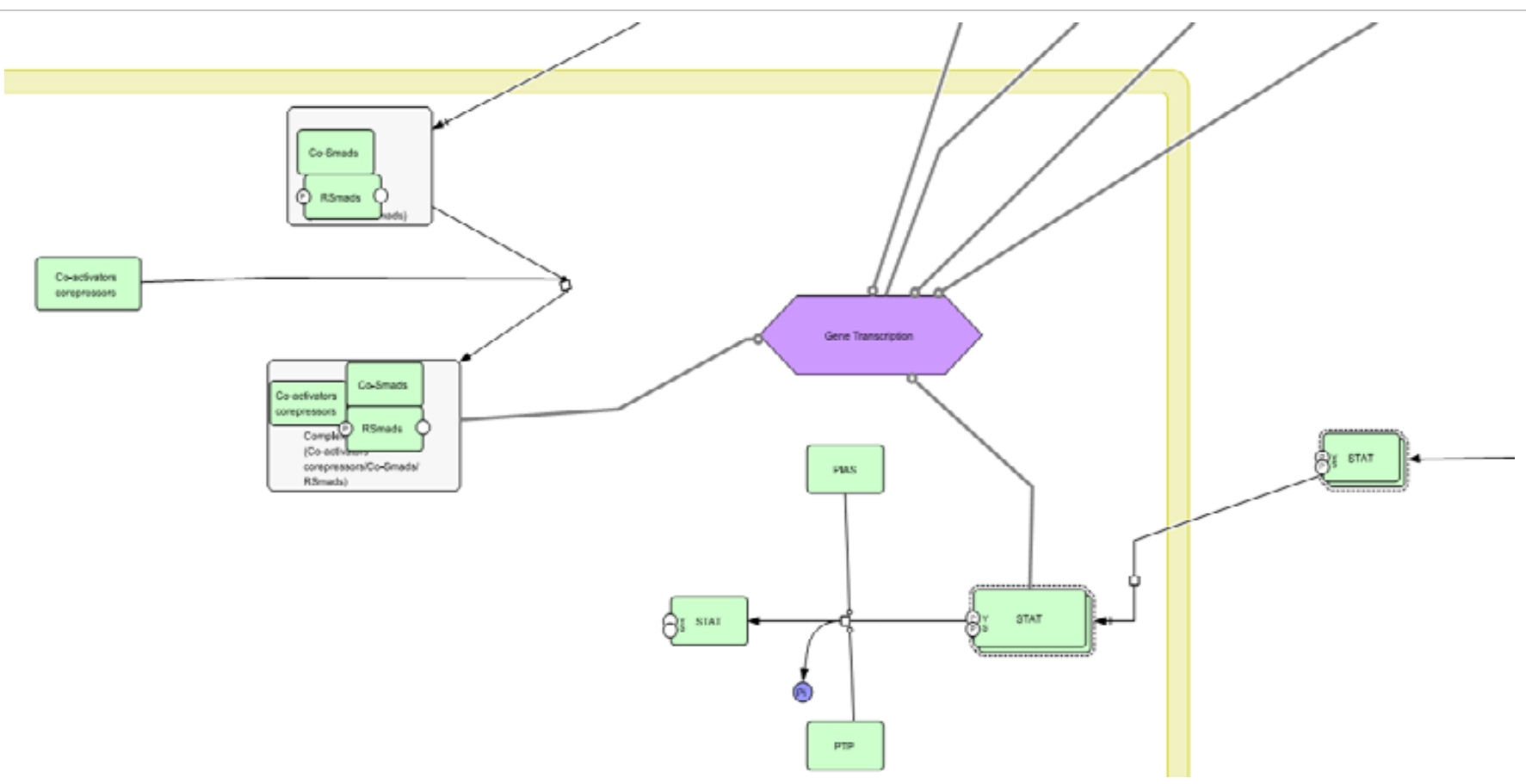

Figure 9c: Crosstalk between JAK-STAT and TGF biological signaling pathways visualized in CellDesigner.

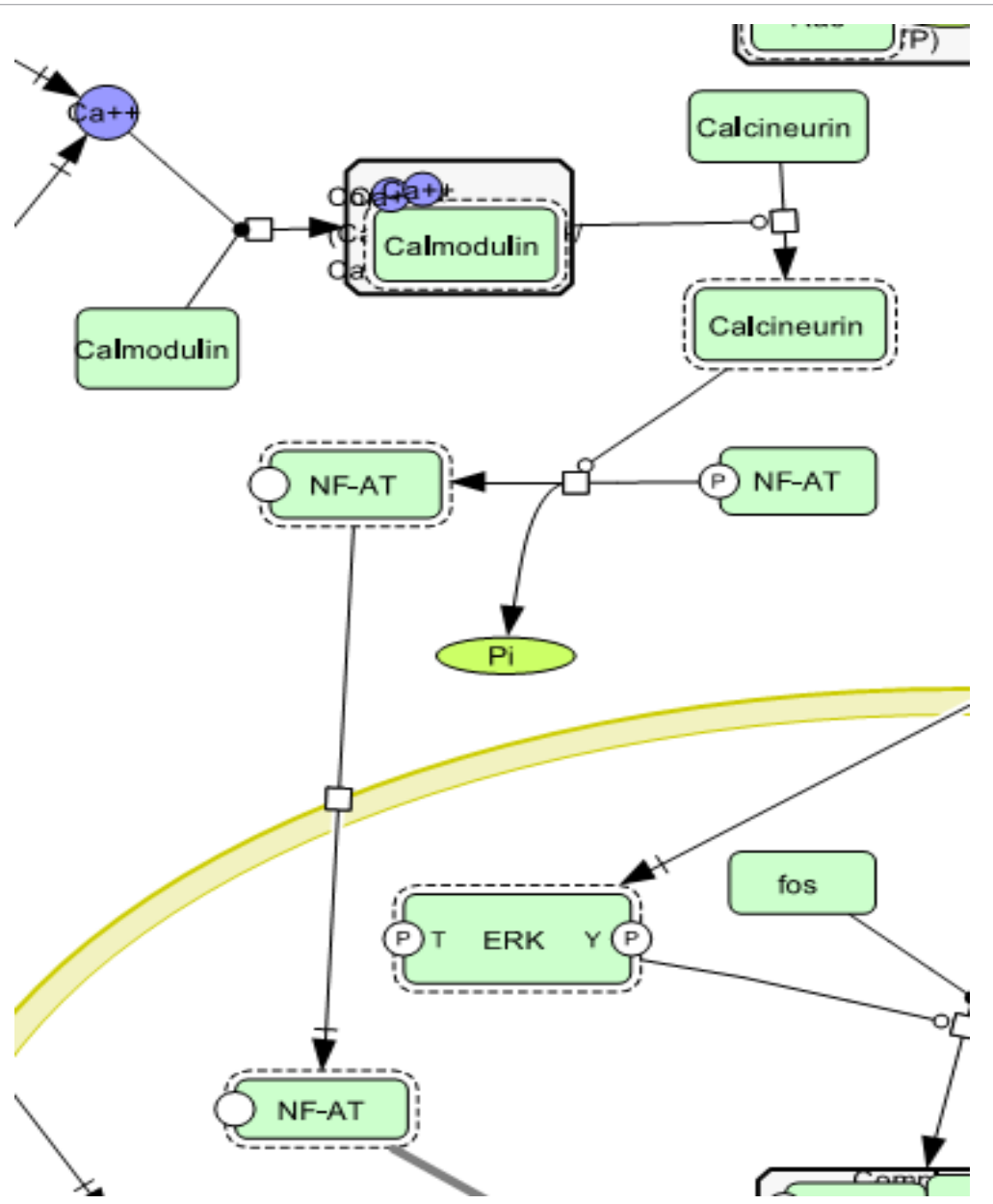

Figure 10a: B-Cell biological signaling pathways visualized in CellDesigner shows activation of NFAT on dephosphorilation mediated by calcineurin 


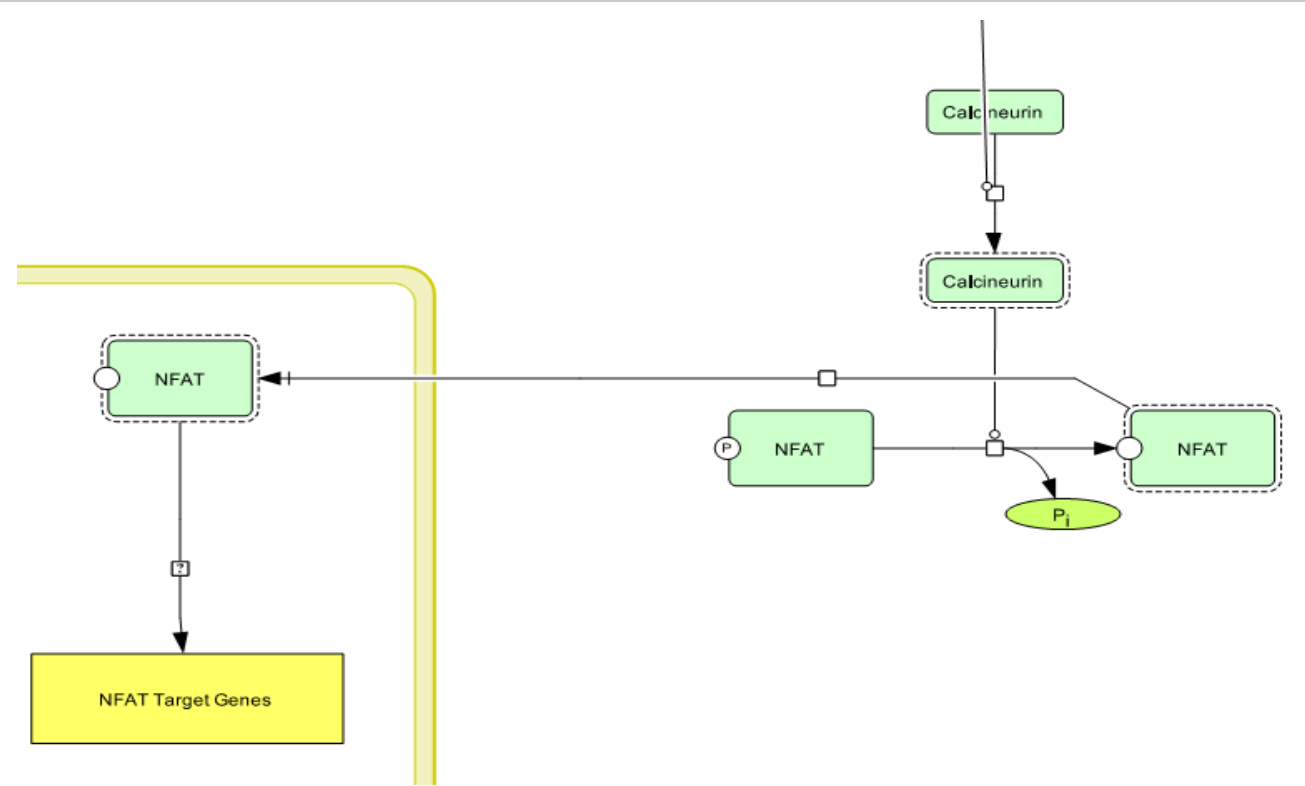

Figure 10b: WNT biological signaling pathways visualized in CellDesigner shows activation of NFAT on dephosphorilation mediated by calcineurin.

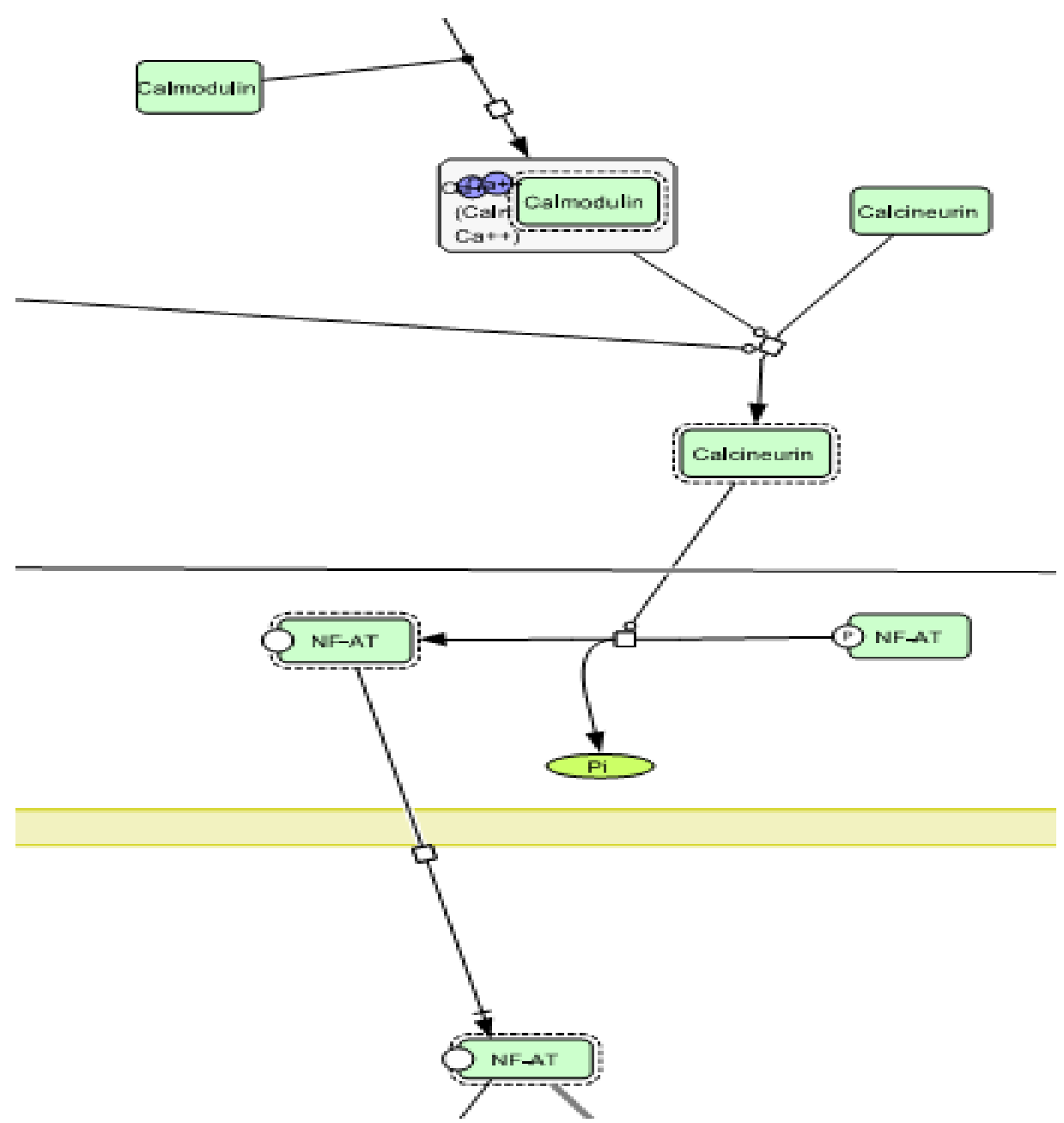

Figure 10c: Crosstalk between B-Cell and WNT biological signaling pathways visualized in CellDesigner. 
tool, pathway models are constructed for all the five signaling pathways and merged signaling pathway network as shown in figures 11-16.

The pathway models simplified the complexity in the analysis of biological pathways. We aimed at the better understanding of the biological signalling pathway by deriving mathematical models for all the pathway models as shown in figures 17-22. The derivation of mathematical models for all the pathways including merged network was followed by the designing of block diagrams or circuit diagrams using the VHDL to describe a logic circuit by function, data flow behavior or structure as shown in figures 23-28. These circuits and mathematical models helped us to calculate the total flux on the nucleus

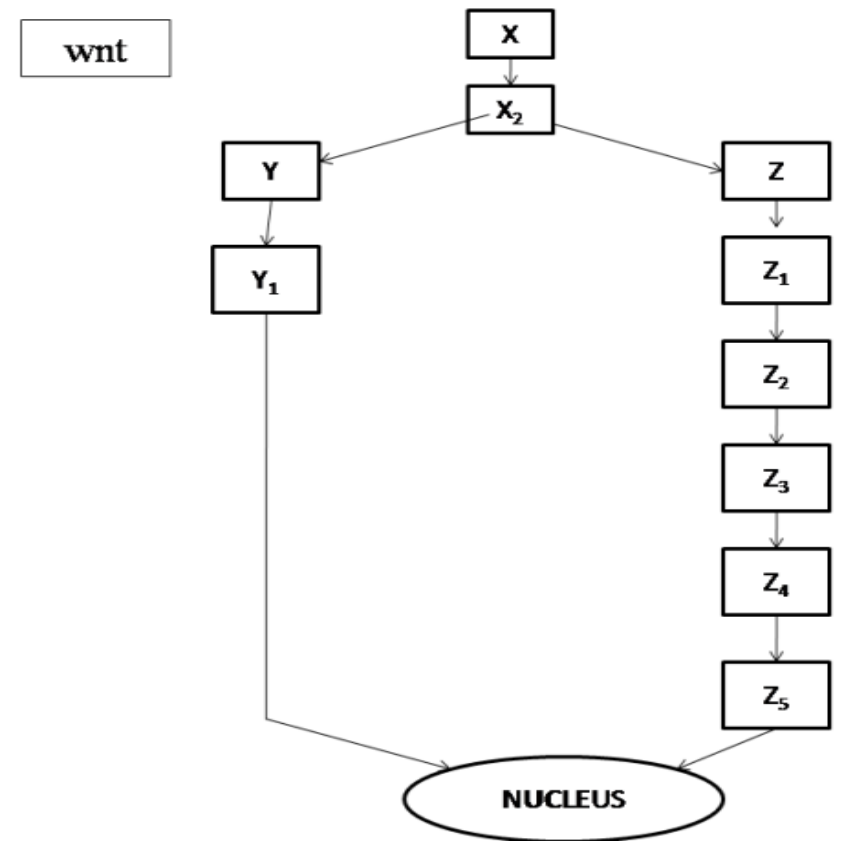

Figure 11: Pathway model for WNT signaling pathway.

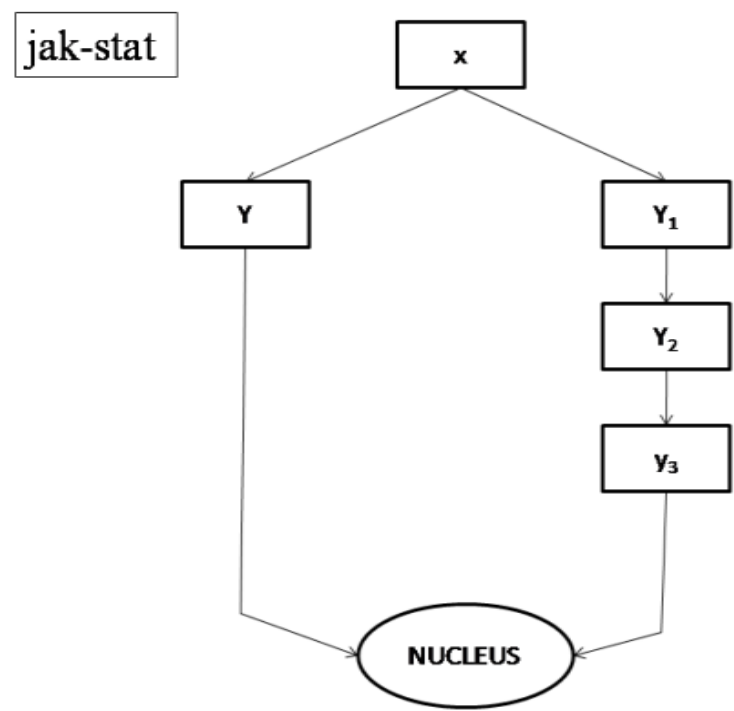

Figure 12: Pathway model for JAK-STAT signaling pathway.

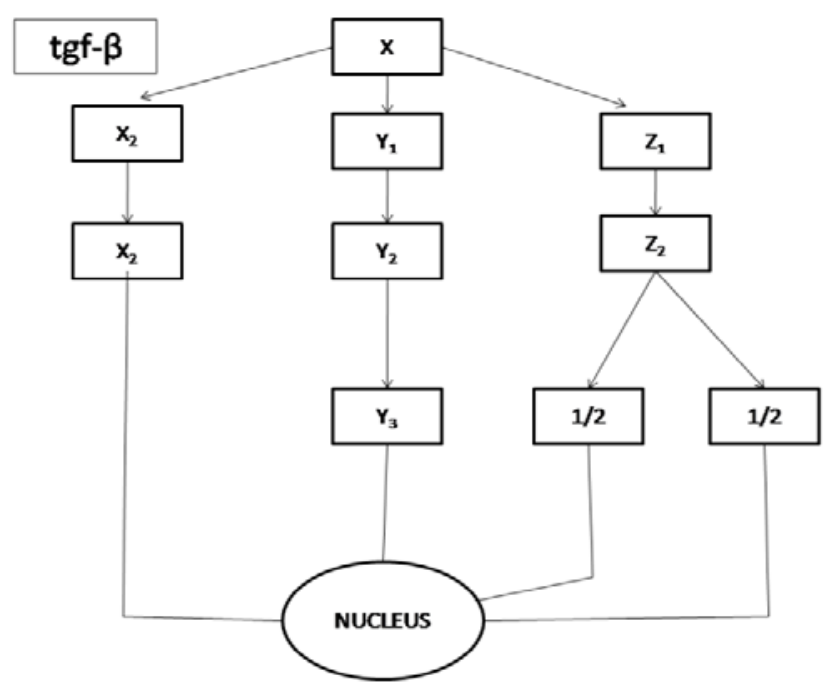

Figure 13: Pathway model for TGF signaling pathway.

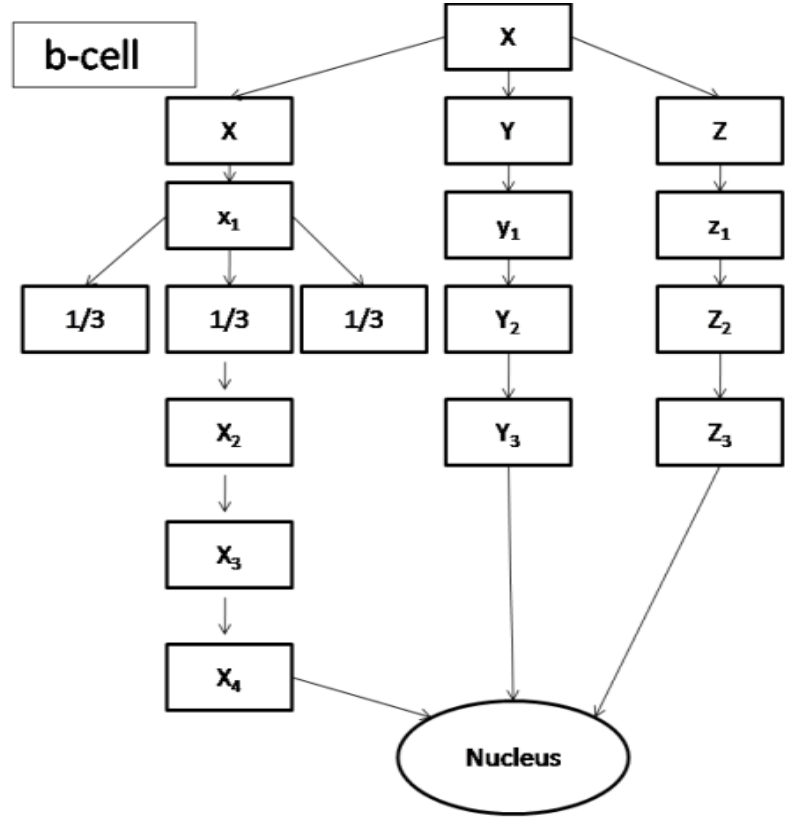

Figure 14: Pathway model for B-Cell signaling pathway.

by deriving the mathematical expressions experiment 1 to experiment 6.

Here input value is considered as ' $\mathrm{X}$ ' and ' $\mathrm{Y}$ ', output value as ' $\mathrm{Z}$ ' in block diagram as shown in figure 23A. The ' $\mathrm{X}$ ' is represented as event of generation of signal and ' $\mathrm{Y}$ ' is represented as signal at part of consideration. The variables 'a' and ' $b$ ' which are involved in number of steps in the activation, finally the activation steps are adding give the output value ' $\mathrm{Z}$ '. In the figure $23 \mathrm{~B}$, the red colour represents the WNT circuit diagram which merged to other block diagram and get total output.

In JAK-STAT block diagram, the input value is taken as ' $\mathrm{X}$ ' and ' $\mathrm{Y}$ ' which connects the variable ' $a$ ' and ' $b$ ', the ' $b$ ' having two activation steps. Both the variables combine to give the output value ' $Z$ ' as shown 


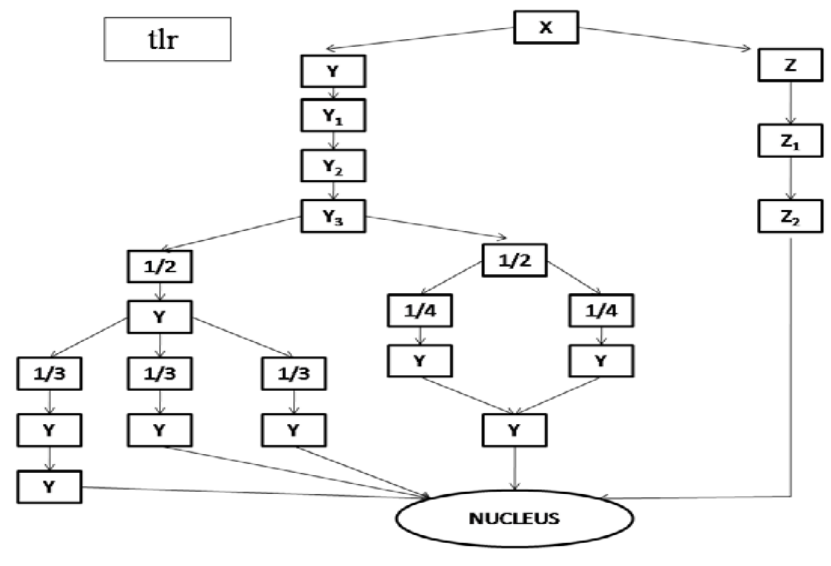

Figure 15: Pathway model for TLR signaling pathway.
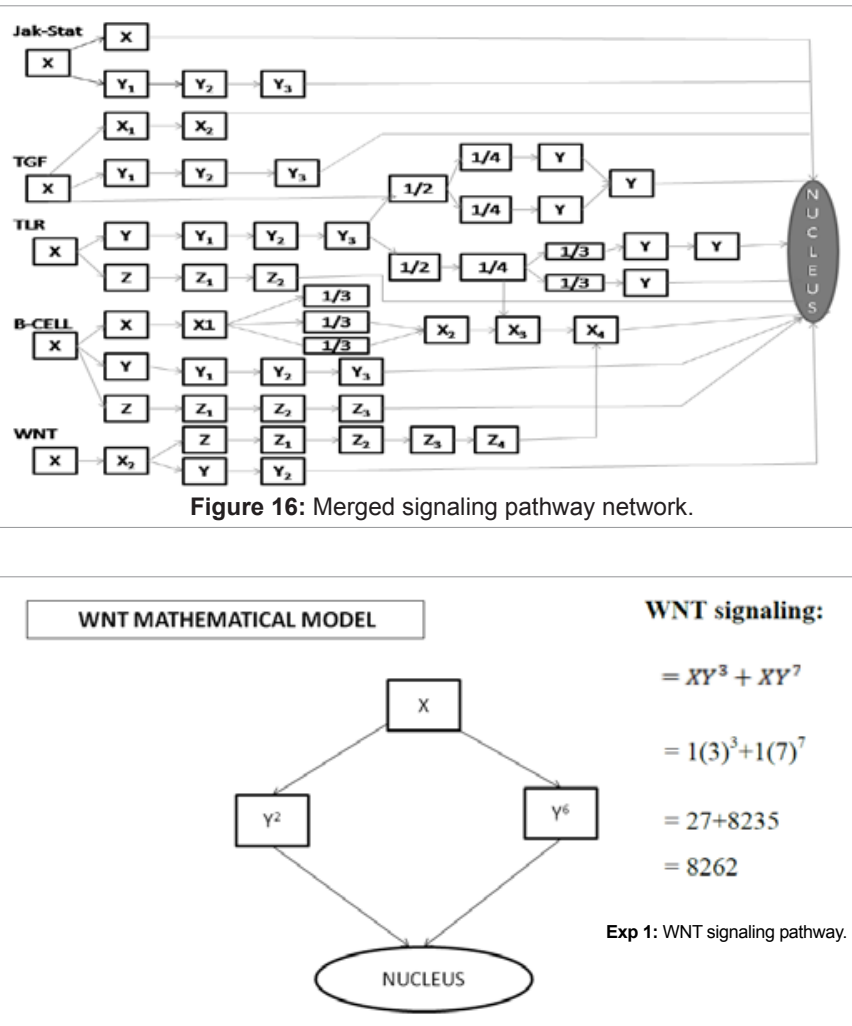

Figure 17: WNT mathematical model.

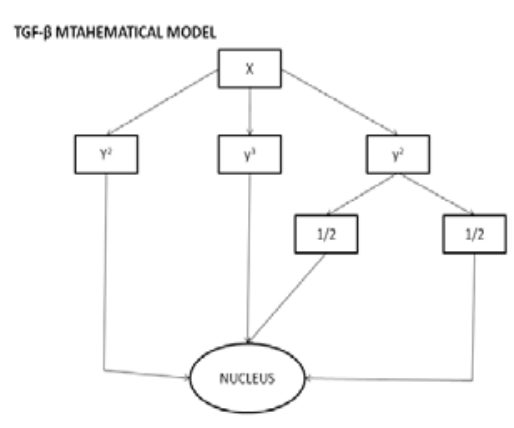

TGF- $\beta$ :

$=X Y^{2}+X Y^{3}+X Y^{3}$

$=1(2)^{2}+1(3)^{3}+1(4)^{3}$

$=4+27+64$

$=95$

Exp 2: TGF- $\beta$ signaling pathway.

Figure 18: TGF-beta mathematical model.
JAK-STAT MATHEMATICAL MODEL

JAK-STAT:

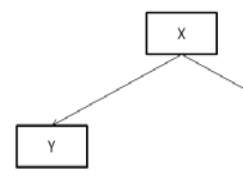

$X Y+X Y^{3}$

$=1(1)+1(3)^{3}$

$=28$

NUCLEUS Exp 3: JAK-STAT signaling pathway.

Figure 19: JAK-STAT mathematical mode.

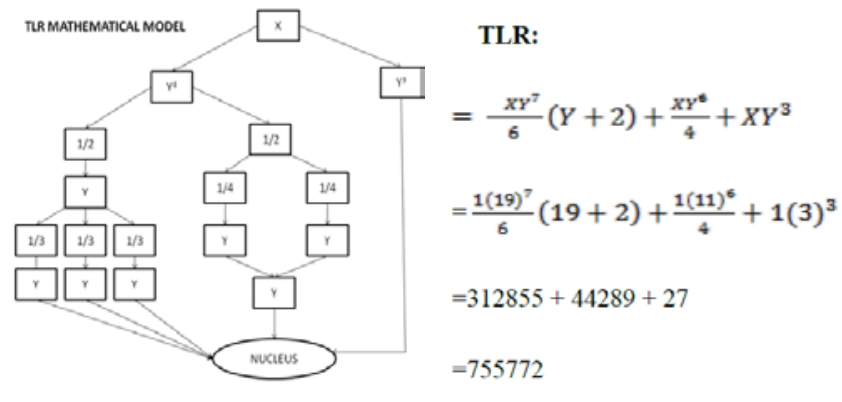

Exp 4: TLR signaling pathway.

Figure 20: TLR mathematical model.

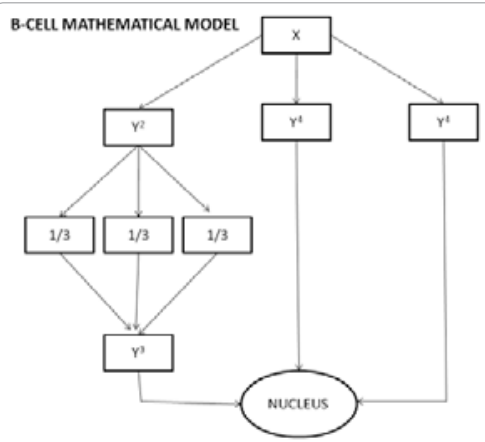

B-cell:

$=X Y^{6}+X Y^{4}+X Y^{4}$

$=1(9)^{6}+1(4)^{4}+1(4)^{4}$

$=38742+256+256$

$=39254$

Exp 5: B-Cell signaling pathway.

Figure 21: B-Cell mathematical model.

in figure 24A. JAK-STAT in red colour indicates its activation in the block diagram which has been designed for merged network as described in figure 24B.

In the B-Cell block diagram, the input value taken as ' $\mathrm{X}$ ' and ' $\mathrm{Y}$ ' connects the variable ' $a$ ', 'b, 'c' and 'd', the 'a' with two activation steps connecting ' $b$ ' and ' $c$ '. Similarly the variable' $d$ ' has four activation steps as shown in figure $25 \mathrm{~A}$. Finally these variables combine to give the output value ' $Z$ '. The block B-cell in red color indicates its activation in the circuit which has been designed for merged network as described in figure 25B.

In TGF block diagram, the input value ' $\mathrm{X}$ ' and ' $\mathrm{Y}$ ' connects to the variables ' $a$ ' ' $b$ ' and 'c', the 'a' and 'b' having number of activation steps, in ' $c$ ' which is diverge into an activation steps. Finally the summation of all the variables gives the output value ' $\mathrm{Z}$ ' as shown in figure 26A. The TGF block highlighted in red colour indicates its activation and it merge with other two blocks and activates all blocks merged in output. 
In TLR block diagram, consider the input value as ' $\mathrm{X}$ ' and ' $\mathrm{Y}$ ', the variables are 'a', 'b', 'c', 'd', 'e', 'f, 'g' , 'h', 'I', 'j', 'k' and 'l'. The 'a' contains three activation steps; 'b' is having two activation steps. One of the activation steps is ' $c$ ', this variable is having tree more activation steps; consider them as variables ' $\mathrm{e}$ ', ' $\mathrm{f}$ ' and ' $\mathrm{g}$ ', then these variables constitutively give ' $j$ '. Another activation step is 'd', this variable divided into two activation step; consider the variable as ' $h$ ' and ' $I$ ', then these variables combine to give ' $k$ '. The variable ' $\mathrm{l}$ ' also contains two activation steps, finally ' $j$ ', ' $k$ ' and 'l' variables on adding gives the output value ' $\mathrm{Z}$ ' as given in figure 27A. The figure $27 \mathrm{~B}$ illustrates that TLR merges with all blocks and activates steps connects to the output ' $\mathrm{Z}$ '.

The input value ' $\mathrm{X}$ ' and ' $\mathrm{Y}$ ' connects to all the five components which are WNT, B-Cell, TLR, TGF and JAK-STAT. These components

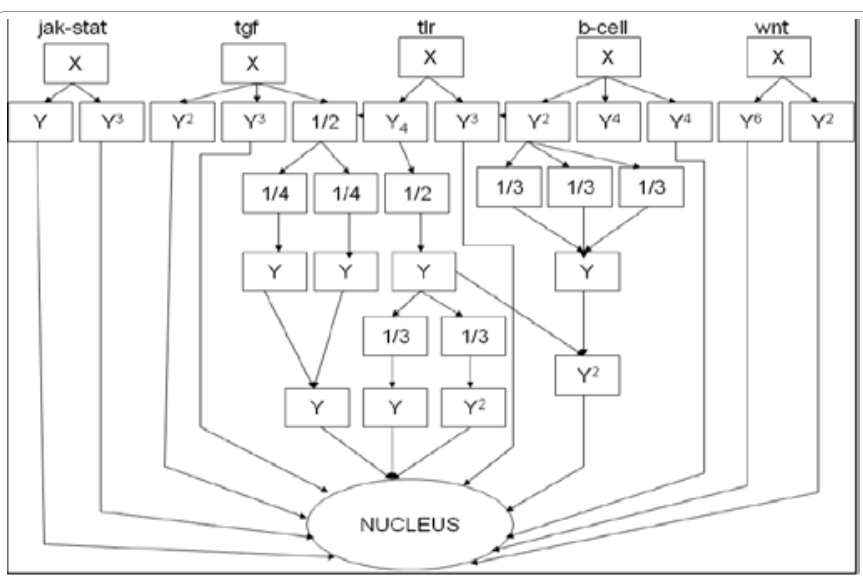

Exp 6: Expression for merged pathway model:

$X Y+X Y^{3}+X Y^{2}+\frac{X Y^{5}}{8}+\frac{X Y^{6}}{6}+\frac{X Y^{7}}{6}+\frac{X Y^{7}}{6}+X Y^{3}+X Y^{3}+X Y^{4}+X Y^{4}+X Y^{6}+X Y^{2}$

$=X Y\left(1+y^{2}\right)+X Y^{2}(1+y)+\frac{X Y^{5}}{8}+\frac{X Y^{6}}{6}+(2 Y+1)+X Y^{3}(2 Y+1)+X Y^{2}\left(Y^{4}+1\right)$

$=4(17)+25(6)+20131+305798+27+343(9)+64(4097)$

$=68+650+325956+3087+262144$

$=591905$

Figure 22: Merged pathway model.
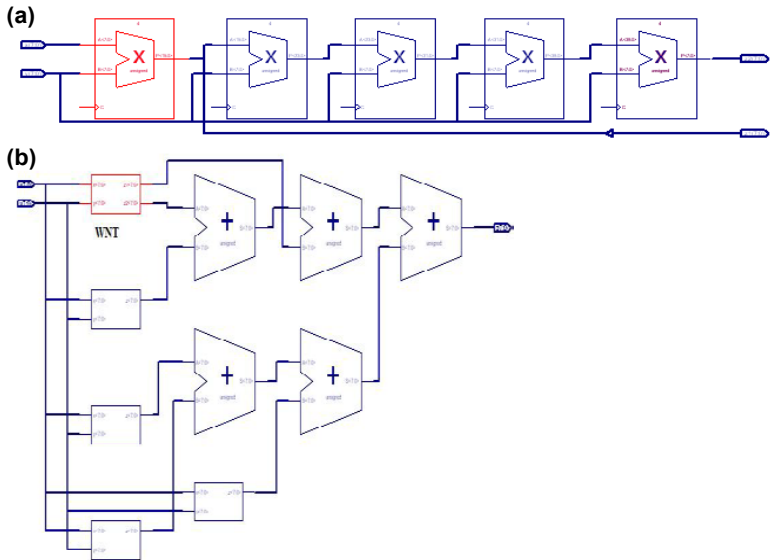

Figure 23: (a) WNT Block diagram; (b) Position of WNT pathway in the merged network.

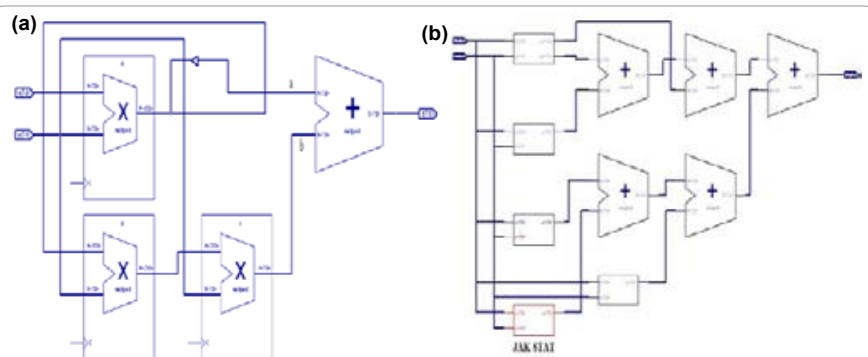

Figure 24: (a) JAK-STAT block diagram; (b) Position of JAK-STAT pathway in the merged network.
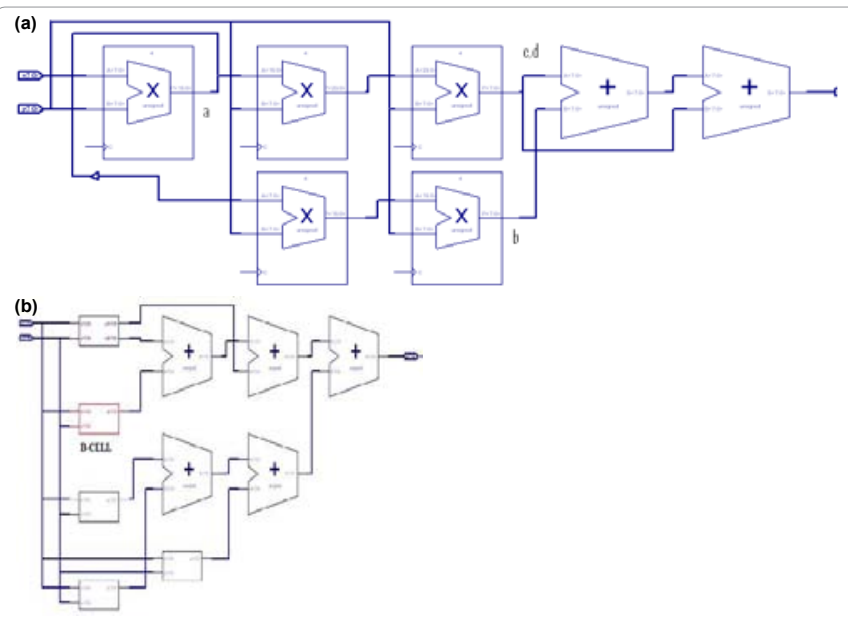

Figure 25: (a) B-Cell block diagram; (b) Position of B-Cell pathway in the merged network.
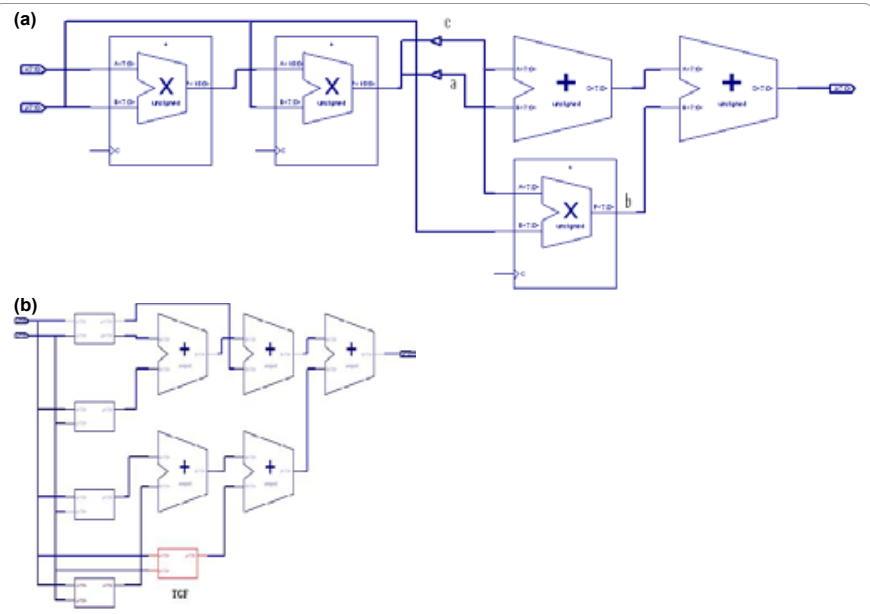

Figure 26: (a) TGF block diagram; (b) Position of TGF pathway in the merged network.

consist of a number of activation steps which are explained in above diagrams. All components are merged and finally give the output value as ' $Z$ ' as shown in figure 28.

\section{Discussion}

The rapidly increasing number of revealed biological pathways calls for the development and use of simulation methods and tools that would facilitate their analysis and virtual experimentation. Metabolic network reconstruction and simulation allows for an in depth insight into 


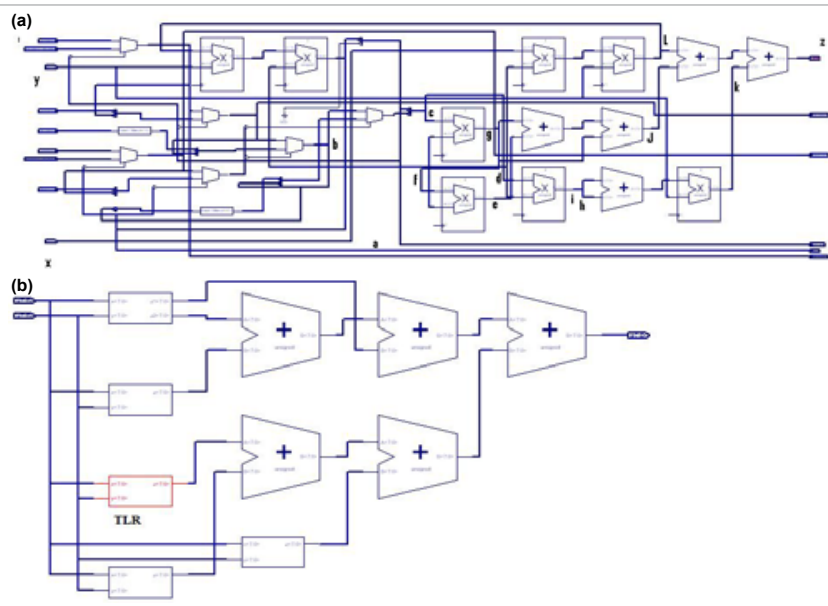

Figure 27: (a) TLR block diagram; (b) Position of TLR pathway in the merged network.

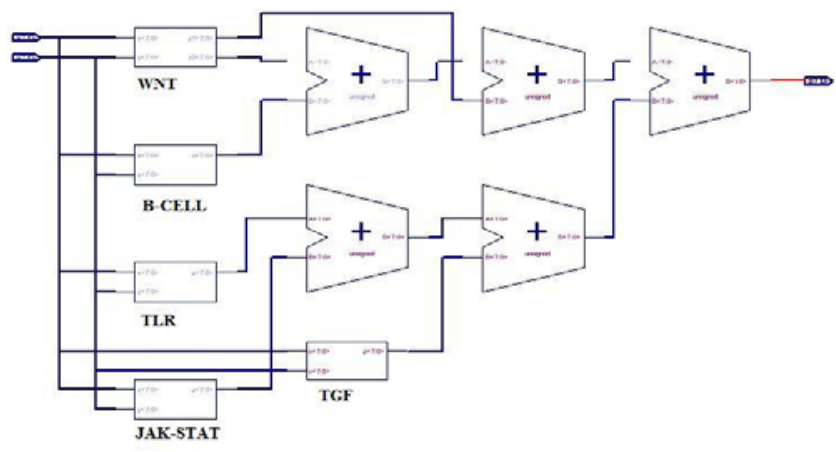

Figure 28: Embed block diagram of all five signaling pathways.

comprehending the molecular mechanisms of a particular organism, especially correlating the genome with molecular physiology [20]. In that concern, the present work for the first time contributes for the representation of engineered models in a consistent format and further simulating them in silico, standardization of representation and model formalism with electronic circuit approaches. Here the knowledge on signaling pathways involved in the clearence/neutralization of endotoxin and the pathways responds for the endotoxin infection is obtained by the manual curation. The pathways were downloaded from the PANTHER pathway database and critically analysed by employing the pathway visualization tool Celldesigner.

A closer assessment of pathways revealed that signaling pathways interact with each other, forming a network. Complexity in pathway network is reduced and common genes were identified in order to reveal the role of all the potential paths in signalling pathway for crosstalk. Group of some kinase enzymes like TAK1, IKK, JKK and MKK3 are commonly absorbed in some pathway interactions. Few pathways shared the same path with in a nucleus. Based on these common genes the initial biological pathway network model in macrophage is successfully designed.

The initial biological pathway models are standardized by constructing the pathway models to reduce the complexity. Pathway models are constructed by assuming the initial protein as $\mathrm{X}$ with its value is 1 and the next coming paths as $\mathrm{x}, \mathrm{y}$ and $\mathrm{z}$, the steps coming under $\mathrm{x}$ paths were labeled as $\mathrm{x}_{1}, \mathrm{x}_{2} \ldots \mathrm{x}_{\mathrm{n}}$ respectively, similarly labeled for $y$ and $z$ paths. Here when the protein gets deviated through different paths, the protein value was divided as per the number of deviating paths. If the value at the protein is 1 and this protein is diverged through two paths, the value was shared as $1 / 2$ to each path, if the value at the protein is 1 and this protein is diverged through three paths, the value was shared as $1 / 3$ to each path. If the value at the protein is $1 / 2$ and this protein is diverged through two paths, the value was shared as $1 / 4$ to each path.

Aimed towards the better understanding of biological signalling pathway, mathematical models for all the pathway models were derived. Here numbers of steps were taken into consideration; if pathway model have single step it used to be labeled as Y, if it is with two steps, it was labeled as Y2 and similarly for three and four steps it was labeled as Y3 and Y4, respectively. Score sharing was same as in construction of pathway models and flux from each model was calculated using derived mathematical expressions.

The derivation of mathematical models for all the pathways including merged network was followed by the designing of block diagrams or circuit diagrams using the VHDL to describe a logic circuit by function, data flow behavior of genes in signal. These circuits and mathematical models helped us to calculate the total flux on the nucleus by deriving the mathematical expressions.

The conclusion methodology we described here will be reliable since it helps in calculating the flux at each node given by a mathematical expression. Also from this method, we establish that the multiple signaling pathways do not have the additive effect but the signals will cancel at the converging points. This method can be applied for any network for modeling and calculating the signal flux from different nodes to nucleus and degree of error depends on pathway modeling. Since it is a manual process, errors in modeling can reflect the method reliability.

\section{Acknowledgement}

All the authors greatly acknowledge University Grant Commission (UGC) for the financial support through major research project [No. 39-280/2010 (SR) Dated 21-12-2010] for the conduction of present work in our laboratory, authors extends warm thanks to The Registrar, Kuvempu University, Jnanasahyadri, Shankaraghatta-577 45, Shivamogga, Karnataka, India for the support and encouragement.

\section{References}

1. Kojima K, Nagasaki M, Miyano S (2010) An efficient biological pathway layout algorithm combining grid-layout and spring embedder for complicated cellular location information. BMC Bioinformatics 11: 335.

2. Schwartz MA, Baron V (1999) Interactions between mitogenic stimuli or a thousand and one connections. Curr Opin Cell Biol 11: 197-202.

3. Ulevitch RJ, Tobias PS (1999) Recognition of gram-negative bacteria and endotoxin by the innate immune system. Curr Opin Immunol 11: 19-22.

4. Robert SM (2006) The Role of Gram-Negative Bacteria. Annu Rev Pathol Mech Dis 1: 467-496.

5. Tzeng YL, Datta A, Kolli VK, Carlson RW, Stephens DS (2002) Endotoxin of Neisseria meningitidis composed only of intact lipid A: inactivation of the meningococcal 3-deoxy-D-manno-octulosonic acid transferase. J Bacteriol 184: $2379-2388$

6. Tsuzuki H, Tani T, Ueyama H, Kodama M (2001) Lipopolysaccharide: neutralization by polymyxin $B$ shuts down the signaling pathway of nuclea factor kappaB in peripheral blood mononuclear cells, even during activation. J Surg Res 100: 127-134.

7. Kacimi R, Giffard RG, Yenari MA (2011) Endotoxin-activated microglia injure brain derived endothelial cells via NF-KB, JAK-STAT and JNK stress kinase pathways. J Inflamm (Lond) 8: 7. 
Citation: Bharath BR, Manjunatha H, Santosh Kumar HS, Bharath K (2013) Non Linear Flux Assessment Model for Endotoxin Neutralizing Pathways. J Comput Sci Syst Biol 6: 057-072. doi:10.4172/jcsb.1000101

8. Hayashi EA Akira S, Nobrega A (2005) Role of TLR in B cell development: signaling through TLR4 promotes B cell maturation and is inhibited by TLR2. J Immunol 174: 6639-6647.

9. Dankesreiter S, Hoess A, Schneider-Mergener J, Wagner H, Miethke T (2000) Synthetic endotoxin-binding peptides block endotoxin-triggered TNF-alpha production by macrophages in vitro and in vivo and prevent endotoxin-mediated toxic shock. J Immunol 164: 4804-4811.

10. Majetschak M, Krehmeier U, Bardenheuer M, Denz C, Quintel M, et al. (2003) Extracellular ubiquitin inhibits the TNF-alpha response to endotoxin in peripheral blood mononuclear cells and regulates endotoxin hyporesponsiveness in critical illness. Blood 101: 1882-1890.

11. Cuschieri J, Billgren J, Maier RV (2006) Phosphatidylcholine-specific phospholipase C (PC-PLC) is required for LPS-mediated macrophage activation through CD14. J Leukoc Biol 80: 407-414.

12. Ajuwon KM, Banz W, Winters TA (2009) Stimulation with peptidoglycan induces interleukin 6 and TLR2 expression and a concomitant downregulation of expression of adiponectin receptors 1 and 2 in 3T3-L1 adipocytes. J Inflamm (Lond) 6: 8.

13. Takeda K, Akira S (2004) TLR signaling pathways. Seminars in Immunology 16: 3-9.
14. Watterson S, Marshall S, Ghazal P (2008) Logic models of pathway biology Drug Discov Today 13: 447-456.

15. Kell DB (2006) Systems biology, metabolic modelling and metabolomics in drug discovery and development. Drug Discov Today 11: 1085-1092.

16. Fell DA (1992) Metabolic control analysis: a survey of its theoretical and experimental development. Biochem J 286: 313-330.

17. Klipp E, Liebermeister W (2006) Mathematical modeling of intracellular signaling pathways. BMC Neurosci 7: S10.

18. Hucka M, Finney A, Sauro HM, Bolouri H, Doyle JC, et al. (2003) The systems biology markup language (SBML): a medium for representation and exchange of biochemical network models. Bioinformatics 19: 524-531.

19. Ogata H, Goto S, Sato K, Fujibuchi W, Bono H, et al. (1999) KEGG: Kyoto Encyclopedia of Genes and Genome. Nucl Acids Res 27: 29-34.

20. Francke C, Siezen RJ, Teusink B (2005) Reconstructing the metabolic network of a bacterium from its genome. Trends Microbiol 13: 550-558. 